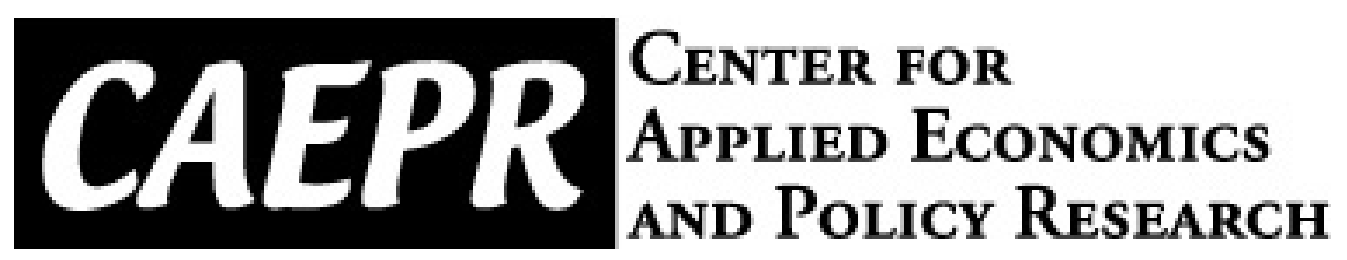

CAEPR Working Paper

\#2008-022

\title{
Strategic Disclosure of Valuable Information within Competitive Environments
}

\author{
Young-Ro Yoon \\ Indiana University Bloomington
}

August 25, 2008

This paper can be downloaded without charge from the Social Science Research Network electronic library at: http://ssrn.com/abstract=1254962.

The Center for Applied Economics and Policy Research resides in the Department of Economics at Indiana University Bloomington. CAEPR can be found on the Internet at:

http://www.indiana.edu/ caepr. CAEPR can be reached via email at caepr@indiana.edu or via phone at 812-855-4050.

(C2008 by Young-Ro Yoon. All rights reserved. Short sections of text, not to exceed two paragraphs, may be quoted without explicit permission provided that full credit, including $\odot$ notice, is given to the source. 


\title{
Strategic Disclosure of Valuable Information within Competitive Environments
}

\author{
Young-Ro Yoon ${ }^{1}$
}

\begin{abstract}
Can valuable information be disclosed intentionally by the informed agent even within a competitive environment? In this article, we bring our interest into the asymmetry in reward and penalty in the payoff structure and explore its effects on the strategic disclosure of valuable information. According to our results, the asymmetry in reward and penalty is a necessary condition for the disclosure of valuable information. This asymmetry also decides which quality of information is revealed for which incentive; if the penalty is larger than the reward or the reward is weakly larger than the penalty, there exists an equilibrium in which only a low quality type of information is revealed, in order to induce imitation. On the other hand, if the reward is sufficiently larger than the penalty, there exist equilibria in which either all types or only high quality type of information is revealed, in order to induce deviation. The evaluation of the equilibrium in terms of expected payoff yields that the equilibrium where valuable information is disclosed strategically dominates the equilibrium where it is concealed.
\end{abstract}

Keywords and Phrases: Strategic information disclosure; Asymmetry in reward and penalty

JEL classification Numbers: D82; M52

\footnotetext{
${ }^{1}$ Department of Economics, Indiana University Bloomington, Address: Wylie Hall Room 211, 100 South Woodlawn, Bloomington, IN 47405, E-mail: yoon6@indiana.edu, Tel) 812-855-8035. I acknowledge Talia Bar, Fwu-Ranq Chang, Sangwook Cho, Roy Gardner, Marco Ottaviani, and Eric Rasmusen for helpful comments. Also I wish to thank conference participants at 2008 Far Eastern Econometric Society Meeting and the 6th Annual International Industrial Organization Conference. I take full responsibility for any error.
} 


\section{Introduction}

"Hope for the best, prepare for the worst"

Frequently, agents compete with one another in a common or similar task and, due to this competitive environment, they are often evaluated based on their performance. While many factors can be considered in these evaluations, one main factor can be his performance in relation to the performances of other competitors. If, in completing a task, an agent was successful but his peers were also successful, he will be given a less positive evaluation than if he alone had been successful, because the reward or the prize would be shared. On the other hand, if an agent was not successful but other competitors also failed, the penalty or the blame would similarly be shared, resulting in a less negative evaluation than if he alone was unsuccessful. As a result of such an evaluation scheme, when carrying out a task each agent must consider not only his own performance but also the performances of other agents with whom he is competing.

Consider one agent carrying out a given task in a competitive environment; if he has meaningful information related to the task, we can predict that he may not want to reveal it to others, in order to be the only agent who completes the task successfully. If information he has is perfect, our prediction may be correct. However, if that information is, although meaningful, not perfect, concealing it from other agents may not be the best strategy, because it could lead the agent in the wrong direction and cause him to be the only agent who fails at the task. In this case, given that the shared failure of the other agents mitigates our agent's failure by sharing the blame under the relative evaluation scheme, revealing his information could produce a better situation than concealing it as he may thus seduce some agents into following him in the wrong direction. This reasoning reveals the possibility that meaningful information could, for this purpose, be revealed even in a competitive environment. If this is the case, then what about the possibility that even high quality information could be revealed? If we only consider an agent with meaningful information, it seems unlikely. However, if we also consider other agents involved in the competitive environment, the answer can be not decisive because other agents' responses to the revealed information must be considered.

Then what characterizes the condition under which valuable information is revealed even in a competitive environment? If it is revealed, which quality of information will be revealed? When and why does the agent with valuable information intend to reveal it? The aim of this paper is to analyze the possibility of strategic information disclosure within competitive environments, by providing the answers to these proposed questions.

The effects of an asymmetry in reward and penalty in the payoff structure on the strategic information disclosure is of particular interest. Sometimes whether the reward or the penalty is stressed can be given objectively. Or agents can interpret the given situation subjectively as one biased toward either reward or penalty via the following reasoning. For example, if a given task is presumed to be relatively easy, then even if the agent successfully completes the task he will earn a relatively small reward because such success was presumed or taken for granted. However, an agent who fails at such an easy task would earn a large penalty because success was strongly anticipated. On the other hand, if a given task is evaluated as being difficult, then even if our agent is unsuccessful, the penalty will not be as large as it would be for the easy task because less is expected of the agent. However, the agent who successfully completes a difficult task may earn a large reward. Following this reasoning, agents can subjectively interpret a easy 
(hard) task as one from which they have less (much) to gain and much (less) to lose. In other words, if the task is evaluated as being relatively easy (hard), then the agent can evaluate her situation subjectively as one in which the payoff structure is biased toward the penalty (reward). ${ }^{2}$ Below it is shown that the asymmetry in reward and penalty, objectively given or subjectively interpreted, is the critical factor in deriving the equilibrium in which valuable information is strategically revealed. The proposed results specifically imply that the strategic information disclosure is related to whether agents hope for the best or prepare for the worst, which is affected by the asymmetry in reward and penalty.

The model with which we deal in this article can be described as follows: Two heterogeneous agents, one partially informed (henceforth $\mathrm{M}$ ) and one uninformed (henceforth $\mathrm{U}$ ) about the unknown true state, compete with each other in the common task of making a forecast about the true state. The signal which M observes and its precision are both private information. $M$ can decide whether to forecast as the leader or delay and forecast simultaneously with $\mathrm{U}$. On the other hand, $\mathrm{U}$ is required to forecast only in round 2. After both players forecast, the true state is revealed and each player earns his payoff according to the correctness of both players' forecasts, under a relative evaluation scheme. Under these set-up, M must decide both when to act (either in round 1 or round 2) and how to act (whether to be truthful in revealing his signal or not). In the case of $U$, his action depends on M's realized timing of forecast because this determines whether meaningful information about true state is observable or not.

The following points describe the main characteristic of the model. First, a waiting option given to the informed agent makes it possible to analyze his strategic decision regarding information disclosure. For example, if an agent expects that others can mimic his action which contains valuable private information, and he is displeased by this fact, he may delay his action if possible as a way to conceal his information. If, on the other hand, he regards other agents' same actions as beneficial, he may prefer to reveal his information rather than conceal it. In this way, controlling the timing of action is a way in which an agent can control the flow of his information. In this model, the waiting option is given only to the informed agent in order to restrict our attention to the strategic revelation of meaningful information. Second, we assume that no cost is imposed for a delay of forecast. This is in order to rule out the possibility that M, despite his desire to delay, avoids doing so due to the cost. Therefore, M's strategic decision on when to forecast can be understood as a voluntary decision derived only from the usefulness of the delay. Third, U has a chance to infer two dimensions (signal and information quality) of M's private information according to M's realized timing of forecast. If $\mathrm{M}$ does not delay and so his forecast is observable, $\mathrm{U}$ has the opportunity to infer M's private signal. Also, the timing of M's forecast itself always gives U a chance to infer the precision of M's signal, which is private information. Hence, how U forms a belief about and infers M's types (signal and information quality) from the available opportunity play key roles in characterizing the equilibrium.

The main results of analysis can be summarized as follows. It is shown that $M$ always reveals his private signal truthfully, regardless of his timing of forecast and the payoff structure. Hence, his decision on the timing of his forecast is no more than a decision regarding the disclosure of his valuable information. Significantly, as there is no cost for a delay, if $\mathrm{M}$ chooses forecast without a delay, this is his voluntary decision and moreover attributable to his desire to intentionally show his private signal to U. However, his

\footnotetext{
${ }^{2}$ For example, think about the penalty kick in a soccer game. From the viewpoint of goalkeeper (kicker), this is a situation in which there is nothing to lose (gain) and much to gain (lose) because the penalty kick is presumed to favor the kicker rather than the goalkeeper; that is, it is assumed that successfully making the goal is relatively easy for the kicker, while preventing the goal is difficult for the goalkeeper.
} 
equilibrium strategies as to when to forecast and which quality of information he reveals vary according to the asymmetry in reward and penalty as follows.

If the penalty is larger than the reward, the unique equilibrium is the partially revealing separating equilibrium in which M's information is revealed only if it is of low quality. M's weak confidence in the correctness of his information, induced by the low quality of information, makes $\mathrm{M}$ concerned about being penalized. Hence, M strategically reveals his information in order to induce U's imitation, which enables him to avoid the risk of being penalized alone. In this equilibrium, $U$ correctly expects that the information thus revealed is of low quality. However, under a payoff structure biased toward the penalty, U also intends to avoid being penalized alone and therefore imitate M's forecast if observable.

On the other hand, if the reward is larger than the penalty, each agent has an incentive to be differentiated from the other, in order to earn the reward alone. However, being differentiated also bears the risk of being penalized alone. Hence what matters is whether the reward is sufficiently large enough to make agents take the risk of being penalized alone. If the reward is weakly larger than the penalty, the concern of being penalized yields a partially revealing separating equilibrium in which M's information is revealed only if it is of low quality; like the situation in which the penalty is stressed, M's action here is motivated by the desire to be imitated. On the other hand, if the reward is sufficiently larger than the penalty, there exists a partially revealing separating equilibrium in which M's information is revealed only if it is of high quality. Moreover, there also exists a pooling equilibrium in which M's information is always revealed regardless of its quality. For both cases, the disclosure of information is an attempt on M's part to induce U's divergence for the sake of earning the reward alone. In the partially revealing separating equilibrium, although $U$ infers that the revealed information is of high quality, he uses a mixed strategy and deviates from M's forecast with a positive probability. In the pooling equilibrium, U always deviates from M's forecast if observable although he knows that it contains valuable information correlated with the true state. These U's equilibrium strategies are based on his incentive to be differentiated, even though he is the one uninformed about the true state; an incentive affected by the payoff structure strongly biased toward the reward.

In this way, the asymmetry in reward and penalty plays a critical role in deciding which quality of information is revealed. The main intuition is as follows: If the stick is stressed, the agents are each biased toward preparing for the worst case in which he is penalized alone. On the other hand, if the carrot is strongly stressed, each agent is biased toward hoping for the best case in which he alone gets the reward. If the carrot is weakly stressed, both are mixed.

In addition, it is shown that if the reward and the penalty are symmetric, the unique equilibrium is the pooling equilibrium in which $\mathrm{M}$ always forecasts after a delay. Therefore his private signal is not revealed at all. In other words, the asymmetry in reward and penalty is a necessary condition for the strategic disclosure of M's private signal. Also, if we consider the case in which the precision of M's signal is completely known, unlike the case in which it is private information, there exists no equilibrium in which M's high-quality information is revealed. This implies that the incomplete information about the precision of M's signal is a necessary condition for the disclosure of M's very precise information.

Although we mainly restrict our attention to the equilibrium in which M's private signal is strategically revealed, there also exists a pooling equilibrium in which M's private signal is not revealed at all. This type of equilibrium is derived if $\mathrm{i}$ ) the reward is greater than the penalty and ii) $\mathrm{U}$ forms a belief regarding M's information quality under which he imitates U's forecast if observable (which is off-the-equilibriumpath). Hence, for the case in which the reward is stressed, the multiple equilibria are derived. As is well 
known, this is due to the fact that any of U's arbitrary beliefs can be assigned for the outcome which never occurs along the equilibrium path. Regarding this, the evaluation of the equilibrium in terms of expected payoff yields that, under all cases, the pooling equilibrium in which M's private signal is not revealed at all is dominated.

The rest of this chapter is organized as follows. Section 2 reviews related literature. Section 3 introduces a model. Section 4 deals with M's truthfulness in revealing his private signal. Section 5 characterizes the equilibrium. In Section 6, we modify the model and go over the cases in which i) the precision of M's signal is public information and ii) the reward and the penalty are symmetric. We also consider the equilibrium in which M's information is not revealed and evaluate the equilibria of all cases in terms of expected payoff. Section 7 concludes.

\section{Related literature}

Conner and Rumelt (1991) and Conner (1995) explicitly mention the possibility of gaining a strategic advantage by being imitated. Both articles propose, using the model of firms' activities in the market, that allowing other firms to imitate or copy can be a dominant strategy if a positive network externality is present. In their models, the network externality, defined exogenously, is positive in the sense that other firms' imitative activities can increase the size of the market; that is, although the pie must be shared between firms exhibiting identical behaviors, if the pie itself grows, thus increasing each firm's slice, imitation can be advantageous. The positive externality defined in our model is different from that of their models in following sense; the externality in this model is positive in that encouraging other's imitative behavior makes it possible for an agent to avoid the risk of being the sole agent penalized. Also, in this model whether the externality is positive or negative is derived endogenously from the payoff structure and the informed agent's information quality.

Poyago-Theotoky (1999), Rosenkranz (2001), Perez-Castrillo and Sandonis (1996), and De Fraja (1993) deal with the topic of information disclosure within competitive environments, although they do so in the contexts different from that of this paper. Poyago-Theotoky (1999) analyzes a non-tournament model of $R \& D$ where firms are engaged in cost-reducing innovation. It is shown that when spillovers of information are treated as endogenous firms never disclose any of their information if their R\&D are chosen non-cooperatively. On the other hand, in our model, we focus on the information disclosure even in the non-cooperative setting. Rosenkranz (2001) considers a setting in which firms first decide on cooperation and then participate either as a joint venture or competitors in an auction. Each firm's dilemma is that while (some) know-how should be revealed in order to determine the possible benefits of cooperation, doing so also opens the firm to the risk of being exploited. According to Rosenkranz (2001), cooperation is a pooling equilibrium strategy if only a little know-how is transferred. It also characterizes the condition under which only the agents with low know-how cooperate. Under similar feature, PerezCastrillo and Sandonis (1996) argue that although the cooperation of R\&D or RJV can be affirmative in the pre-competitive state of $R \& D$, the spillover of technology or know-how of this stage can be negative later because it can induce a more competitive situation in the product market. However, the main focus of their model is to find the existence of incentive contracts that induce firms to share their know-how in RJVs facing technical uncertainty. De Fraja (1993) considers the setting in which a firm can have an incentive to reveal knowledge to its rival during the patent race. In his model, although the rival's success may be unfavorable in short-term, if there are the product market benefits as its consequence, a 
firm may intend to help a rival by revealing knowledge. In this sense, it shares the similar feature with Conner and Remelt (1991) and Conner (1995).

The topic of information sharing has been analyzed widely in depth in I.O. literature, in the context of information sharing in an oligopoly market. For literature about information sharing in an oligopoly market, see Shapiro (1973), Clarke (1983), Fried (1984), Vives (1984, 1990), Gal-Or (1985, 1986), Li (1985), Raith (1996), and Creane (1998). ${ }^{3}$ In general, this literature explores the conditions under which firms benefit from sharing information and the incentives under which firms commit to the rule of information sharing. ${ }^{4}$ Although this literature is relevant to the model discussed in this paper, information sharing between agents is not the main topic of this paper because only one agent is privately informed. Instead our model focuses on the informed agent's strategic incentive to reveal meaningful information. Also, we de not address the topic of commitment. Whether to reveal private information or not is determined by the informed agent's private incentive in the setting of non-cooperative competition. In addition, much of the literature assumes that information is revealed truthfully by assuming that an agency is responsible for transferring the information correctly or that the information is verifiable. ${ }^{5}$ In our model, the truthfulness of the revealed information is not assumed but derived endogenously.

Gul and Lundholm (1995), Frisell (2003) and Mailath (1993) use the notion, in their analysis, that the timing of action reveals private information. In Gul and Lundholm (1995) and Frisell (2003), each agent infers the precision of the other agent's signal from the timing of action. Both models consider only the fully separating equilibrium and derive the equilibrium of the endogenous timing of action. ${ }^{6}$ The main contribution of Gul and Lundholm (1995) is to show that the phenomenon of informational cascade can happen endogenously even when all players' information is used efficiently, if only the informational externality matters. Frisell (2003) characterizes the equilibrium of the endogenous timing of actions using a model in which two firms select a product design when its true value is not known. The results propose that if the payoff externality is positive or relatively weakly negative, the more-informed firm acts as the leader. On the other hand, if the payoff externality is strongly negative, the more-informed firm acts as the follower because it has more to gain from outwaiting the other firm. Although the setting is a bit different, our model proposes an equilibrium in which the (more) informed firm acts as the leader despite the fact that the payoff externality is strongly negative. This result is based on the observation that the revealed information can induce the other agent to deviate from it, which is not considered in Frisell (2003). Mailath (1993) addresses the topic of market entrance using set-up similar to that of this model in that only one informed firm has a waiting option and the uninformed firm does not. In the unique stable equilibrium, the informed firm operates as the leader although its ex-ante profit is higher when it produces simultaneously with the uninformed firm. The intuitive explanation is that the informed firm's delay reveals the private information that the market is profitable even though the firm would like to conceal it by delaying. In his model, as the precision of the informed firm's information is assumed to be perfect, how the quality of information matters is not considered. In our model, on the other hand, when

\footnotetext{
${ }^{3}$ Vives (1990), Raith (1996) and Creane (1998), especially, provide a good survey of this issue.

${ }^{4}$ Usually it is assumed that firms commit to share information truthfully before they actually observe their private signals.

${ }^{5}$ Unlike other papers in the literature, Ziv (1993) addresses the topic of truthfulness in information revelation. It attempts to endogenize the incentives for truthful information sharing in an oligopoly by providing a mechanism under which the truth-telling of private information is in its own best interest, despite the fact that verification is impossible.

${ }^{6}$ It is assumed that there exists one-to-one mapping such that the observation of timing of action reveals the precision (variance) of the signal perfectly. Also as the variances are drawn from the nonatomic distribution and only the symmetric equilibrium is dealt with, the possibility of the simultaneous action by both agents is not considered.
} 
the informed agent uses a waiting option, his private signal about the true state cannot be inferred. The uninformed agent can only infer the quality of the private signal from the informed agent's delay.

Okuno-Fujiwara, Postlewaite and Suzumura (1990) provides the sufficient condition under which there is a complete revelation of private information (the so-called unraveling result). In their model, what the privately informed agents decide is whether to reveal some or all of their information to other agents. That is, the decision is about the optimal amount of information they share with other agents. In our model, what the informed agent decides is the quality of information to disclose, not the amount of information. Milgrom (1981) and Milgrom and Roberts (1986) show the possibility that information sharing can be derived endogenously from a strategic choice, not from the setting of the commitment. When it is known that an agent is privately informed, if the information is verifiable and the information disclosure is not costly, all private information is revealed (whether it is the favorable choice or not) because the unfavorable information cannot be concealed. ${ }^{7}$ In our model, whether information is favorable or not is based not on its contents but on the quality of information. Hence, in our model the so-called unravelling equilibrium can be defined as one in which private information is revealed endogenously regardless of its quality.

Broadly speaking, the literature regarding herding and anti-herding can be said to be related to the model addressed in this article. ${ }^{8}$ (For examples of this literature, see Banerjee (1992), Bikhchandani, Hirshleifer, and Welch (1992), Scharfstein and Stein (1992), Trueman (1994), Avery and Chevalier (1999), and Effinger and Polborn (2001).) This literature usually assumes that the ordering of action is given exogenously and analyzes the subsequent players' responses against the exogenously revealed prevailing information. What we are interested in, on the other hand, is how the information which the subsequent player can make use of is generated endogenously from strategic reasoning, while considering the subsequent player's response to the revealed information.

\section{Model}

Suppose there are two agents $i \in\{M, U\}$ whose jobs are to provide a forecast about the unknown true state of a forthcoming period. These two agents are the agencies which work independently in the forecasting industry. ${ }^{9}$ The true state is $w \in\{H, L\}$ and these two states are mutually exclusive. The prior probability of each state is $\operatorname{Pr}(w=H)=\operatorname{Pr}(w=L)=\frac{1}{2}$. Before making a forecast, one agent has the opportunity to observe his own signal $\theta \in \Theta=\{h, l\}$ which is correlated with the true state. The other agent has no chance to observe any informative signal correlated with the true state. Throughout this paper, we denote the agent who can observe $\theta$ as $\mathrm{M}$ (informed agent), and the other agent who

\footnotetext{
${ }^{7}$ If information is concealed, other agents can perfectly infer that information is unfavorable and therefore the privately informed one wants to conceal it. This reasoning is similar to that through which, in Mailath (1993), the informed firm acts as the leader in the unique stable equilibrium.

${ }^{8}$ However, in our model as the agent who responds to the revealed information is the uninformed one, the conventional meaning of herding or anti-herding, which is the disregard of private information, does not happen. Instead, imitation and deviation from the prevailing information are derived. Also the reputation effect which is usually considered in the literature addressing herding and anti-herding is not dealt with explicitly in this paper.

${ }^{9}$ We want to distinguish our case from those in which the agents are hired by one principal and the discussion regarding the optimal contract provided by the principal can be the main topic. We adopt the relative evaluation scheme as given and focus on the topic of the strategic information disclosure under competitive environment characterized by relative evaluation scheme. This also makes the model free from subordinate concerns, such as why the uninformed agent is hired by the principal.
} 
cannot as $\mathrm{U}$ (uninformed agent). It is assumed that $\theta \in\{h, l\}$ is private information, so that $\mathrm{U}$ does not know which signal is observed by $\mathrm{M}$.

The signal $\theta$ partially reveals information about the true state in following manner: $\operatorname{Pr}(\theta=h \mid w=$ $H)=\operatorname{Pr}(\theta=l \mid w=L)=p$ and $\operatorname{Pr}(\theta=h \mid w=L)=\operatorname{Pr}(\theta=l \mid w=H)=1-p$ where $p \in\left(\frac{1}{2}, 1\right)$. Here, $p$ measures the precision of M's signal $\theta$, so that it can also be interpreted as the quality of information. As $p$ approaches $\frac{1}{2}$, it means that his signal becomes less informative and as $p$ approaches 1 , it means that his signal becomes more informative about the true state. We assume that $p$ is private information and it is drawn from the uniform distribution $Z(p)$ where $p \in\left(\frac{1}{2}, 1\right)=P$.

M's action set is denoted by $\left\{a_{m}, t_{m}\right\}$. Here, $a_{m} \in F=\{h, l\}$ denotes M's forecast. If $a_{m}=h$ $\left(a_{m}=l\right)$, it denotes that M's forecast is $w=H(w=L)$. M has two rounds during which he can forecast; this forecast is irreversible and $t_{m} \in T=\left\{t_{1}, t_{2}\right\}$ denotes M's timing of forecast where $t_{1}\left(t_{2}\right)$ denotes round 1 (round 2). M can decide when to forecast endogenously. If he forecasts in round 1 (round 2), then it is denoted by $t_{m}=t_{1}\left(t_{m}=t_{2}\right)$. Although $\mathrm{M}$ delays his forecast, no cost is imposed for his delay. ${ }^{10}$ On the other hand, $\mathrm{U}$ is required to forecast only in round 2. Hence, U's action set is $A_{u}=\left\{a_{u}\right\}$ where $a_{u} \in F=\{h, l\}$.

If $t_{m}=t_{1}$, that is, if both agents forecast sequentially, $\mathrm{U}$ can observe M's forecast before announcing his own forecast. On the other hand, if $t_{m}=t_{2}$, that is if both agents forecast simultaneously, $\mathrm{U}$ has no chance to observe M's forecast. In this case, $\mathrm{U}$ knows that $\mathrm{M}$ did not take the opportunity to forecast in round 1 .

Each agent's ex-post payoff, which is given by the market, is determined after the realization of the true state $w$ and is conditional on the correctness of both agents' forecasts, $a_{i}$ and $a_{-i}$, as follows:

\begin{tabular}{|c|c|c|}
\hline$w$ & $a_{u}=w$ & $a_{u} \neq w$ \\
\hline$a_{m}=w$ & 1,1 & $\gamma,-\phi$ \\
\hline$a_{m} \neq w$ & $-\phi, \gamma$ & $-1,-1$ \\
\hline
\end{tabular}

\section{Table 1: Ex-post payoff structure, $\gamma>1$ and $\phi>1$}

We assume that market does not know which agent is informed and not informed and therefore considers only the correctness of both agents' finalized forecasts in evaluating the agents. The payoffs assigned when the forecast turns out to be correct, i.e., $a_{i}=w$, can be interpreted as a reward or prize and that given when it turns out to be wrong, i.e., $a_{i} \neq w$, can be interpreted as a penalty or blame given by the market.

This payoff structure is designed to incorporate the competitive environment two agents face. Suppose that both agents forecasted identically. Then if their forecasts reveal the true state correctly, both earn +1 and, if not, both earn -1 . On the other hand, if both agents' forecasts are different, the agent who made a correct forecast gets $\gamma>1$ and the agent who made a false forecast gets $-\phi<-1$. In other words, if an agent's forecast turns out to be correct, the other agent's identical forecast causes a negative externality because the reward must be divided. On the other hand, if an agent's forecast turns out to

\footnotetext{
${ }^{10}$ Although we assume that a delay cost is imposed for his delay, under the given model, the additional result we can derive is that $\mathrm{M}$ would not delay his forecast if a delay cost is greater than his expected gain from a delay even in the case where a delay is beneficial. If a delay is not beneficial, a delay cost would not cause any change.
} 
be wrong, the other agent's identical forecast causes a positive externality because the penalty will be shared. However, as the true state is revealed only after both agents forecast, whether $a_{i}=w$ or $a_{i} \neq w$ is not verified in advance and therefore an uncertainty is embedded. For the majority of the analysis, we assume that $\gamma \neq \phi .{ }^{11}$ Hence, our case is either $\gamma>\phi$ or $\gamma<\phi$ where the first (second) denotes the case in which the payoff structure is biased toward the reward (penalty). Through this approach, we can analyze the effects of asymmetry in reward and penalty on each player's strategic behavior.

The timing of the game is described as follows:

T1) Nature chooses $\theta \in\{h, l\}$ and $p \in\left(\frac{1}{2}, 1\right)$. M observes both $\theta$ and $p$. The ex-post payoff structure is announced.

T2) Before round 1 starts, $M$ decides when to forecast (either in round 1 or in round 2, but not in both) and whether to be truthful in revealing $\theta$ or not.

T3) Round 1 starts. M forecasts if he decided to do so. If not, he waits until round 2 starts.

T4) Round 2 starts. If $\mathrm{M}$ forecasted in round $1, \mathrm{U}$ observes $a_{m}$ and forecasts as the follower. If not, both $\mathrm{M}$ and $\mathrm{U}$ forecast simultaneously.

T5) After two rounds are over, the true state $w$ is revealed and each player earns his payoff following the ex-post payoff structure.

A pure strategy for $\mathrm{M}$ is a pair of functions $\sigma_{m}=\left(\sigma_{m 1}, \sigma_{m 2}\right)$ where i) $\sigma_{m 1}: \Theta \times P \longrightarrow T$ is the choice regarding the timing of forecast as a function of $\theta$ and $p$, and ii) $\sigma_{m 2}: \Theta \times P \times T \longrightarrow F$ is M's choice of forecast as a function of $\theta, p$ and $t_{m}$. The pure strategy for $\mathrm{U}$ is $\sigma_{u}=\left(\sigma_{u 1}, \sigma_{u 2}\right)$ where $\sigma_{u 1}: a_{m} \longrightarrow a_{u}$ is U's forecast as a function of M's forecast when $t_{m}=t_{1}$ (when $a_{m}$ is observable) and $\sigma_{u 2}$ is a forecast when $t_{m}=t_{2}$ (when $a_{m}$ is not observable). We also allow a mixed strategy for both players.

This model uses the Perfect Bayesian equilibrium concept. Within the model, there exist two types private information, $\theta \in\{h, l\}$ and $p \in\left(\frac{1}{2}, 1\right)$. In the case of $\mathrm{U}$, he will have the chance to infer $\theta$ only if $t_{m}=t_{1}$. In this case, as $a_{m}$ is observable, $\mathrm{U}$ forms a belief with regard to whether $\mathrm{M}$ is truthful in revealing $\theta$ or not. Also, $\mathrm{U}$ can always observe M's timing of forecast which conveys information about $p$. That is, after observing $t_{m} \in\left\{t_{1}, t_{2}\right\}$, U forms a belief regarding $p$. Let $\lambda\left(a_{m}=\theta\right)$ be U's belief for the truthfulness of M's action and $Z\left(p \mid t_{m}\right)$ be a posterior belief over $p$. Then, each agent's strategy $\sigma_{m}$, $\sigma_{u}$ and $\lambda\left(\theta=a_{m}\right), Z\left(p \mid t_{m}\right)$ constitute a PBE if each agent's ex-ante expected payoffs are maximized for given beliefs $\lambda\left(\theta=a_{m}\right), Z\left(p \mid t_{m}\right)$, and the other agent's strategy. Also, in equilibrium, $\lambda\left(\theta=a_{m}\right)$ and $Z\left(p \mid t_{m}\right)$ should be consistent with $\sigma_{m}$ in the Bayesian sense. This can be formalized as follows:

\section{Definition 1}

A Perfect Bayesian Equilibrium is a pair of strategies for $M$ and $U, \sigma_{m}^{*}=\left(\sigma_{m 1}^{*}(p, \theta), \sigma_{m 2}^{*}\left(p, \theta, t_{m}\right)\right)$, $\sigma_{u}^{*}=\left(\sigma_{u 1}^{*}\left(t_{m}=t_{1}, a_{m}\right), \sigma_{u 2}^{*}\left(t_{m}=t_{2}\right)\right)$ and beliefs of $U, \lambda\left(a_{m}=\theta\right)$ and $Z\left(p \mid t_{m}\right)$, such that:

1)

$$
\sigma_{m}^{*} \in \arg \max _{t_{m} \in\left\{t_{1}, t_{2}\right\}, a_{m} \in\{h, l\}} \pi_{m}\left(t_{m}, a_{m}, \sigma_{u}^{*}, p, \theta, w\right) \text { for all } p \text { and } \theta
$$

2) The beliefs of $U, \lambda\left(a_{m}=\theta\right)$ and $Z\left(p \mid t_{m}\right)$, are derived from $\sigma_{m}^{*}(\cdot)$, the prior beliefs, $a_{m}$ if observable, and $t_{m}$, using Bayes's rule whenever applicable.

\footnotetext{
${ }^{11}$ In section 6.2 , we go over the case in which the reward and the penalty are symmetric for comparison.
} 
3)

$$
\sigma_{u 1}^{*} \in \arg \max _{a_{u} \in\{h, l\}} \int \pi_{u}\left(a_{u}, w, a_{m}, p ; t_{m}=t_{1}\right) d Z\left(p \mid t_{1}\right)
$$

where

$$
\pi_{u}(\cdot)=\lambda(\cdot) \sum_{w \in\{H, L\}} \operatorname{Pr}\left(w \mid \theta=a_{m}\right) \pi_{u}\left(a_{m}, a_{u}, w\right)+(1-\lambda(\cdot)) \sum_{w \in\{H, L\}} \operatorname{Pr}\left(w \mid \theta \neq a_{m}\right) \pi_{u}\left(a_{m}, a_{u}, w\right)
$$

4)

$$
\sigma_{u 2}^{*} \in \arg \max _{a_{u} \in\{h, l\}} \int \pi_{u}\left(a_{u}, w, p ; t_{m}=t_{2}\right) d Z\left(p \mid t_{2}\right)
$$

where

$\pi_{u}(\cdot)=\lambda(\cdot) \sum_{w \in\{H, L\}} \sum_{\theta \in\{h, l\}} \operatorname{Pr}(w, \theta) \pi_{u}\left(a_{m}=\theta, w, \cdot\right)+(1-\lambda(\cdot)) \sum_{w \in\{H, L\}} \sum_{\theta \in\{h, l\}} \operatorname{Pr}(w, \theta) \pi_{u}\left(a_{m} \neq \theta, w, \cdot\right)$

\section{Truthfulness in revealing $\theta$}

Note that M's signal $\theta \in\{h, l\}$ is private information. As U can observe only the announced forecast $a_{m}$, $\mathrm{M}$ can make a strategic decision on whether to be truthful in revealing $\theta$ or not. Here, M's truthfulness in revealing $\theta$ is important because it determines whether or not M's true signal $\theta$ can be inferred perfectly from observing $a_{m}$, which affects U's best response.

In this section, we show that, in regard to the truthfulness in revealing $\theta$, M's strategy to deviate from $\theta$ is strictly dominated regardless of $t_{m} \in\left\{t_{1}, t_{2}\right\}$. That is, $\mathrm{M}$ always reveals $\theta$ truthfully. ${ }^{12}$ This also implies that in equilibrium $\mathrm{U}$ gives zero weight to the possibility that $a_{m} \neq \theta$ regardless of $t_{m} \in\left\{t_{1}, t_{2}\right\}$. This result simplifies the analysis which will be discussed in the subsequent sections.

First, consider the case in which $\mathrm{M}$ forecasts in round 1. In this case, from the following reasoning, it can be checked that a pooling strategy cannot constitute an equilibrium. As M's type is $\theta \in\{h, l\}$, M's pooling strategy is either " $a_{m}=h$ whether $\theta=h$ or $\theta=l$." or " $a_{m}=l$ whether $\theta=h$ or $\theta=l$.". Note that whether $\theta=h$ or $\theta=l$, both cases are ex-ante symmetric to M. Hence, for each of U's strategies, the ex-ante expected payoff from being truthful when $\theta_{A}=h$ should be the same as that for the case in which $\theta_{A}=l$. Also, the ex-ante expected payoff from telling a lie when $\theta_{A}=h$ should be the same as that of the case in which $\theta_{A}=l$. Hence, if being truthful (or deviating from $\theta$ ) is A's best response when $\theta_{A}=h$, it should be the same when $\theta_{A}=l$. Then, obviously M's pooling strategy, " $a_{m}=h$ whether $\theta=h$ or $\theta=l$ " or " $a_{m}=l$ whether $\theta=h$ or $\theta=l$ ", cannot constitute an equilibrium because it means that sometimes being truthful is better and sometimes telling a lie is better, according to the given private signal $\theta \in\{h, l\}$ although it is ex-ante symmetric. Hence, a pooling strategy cannot constitute the equilibrium.

\section{Lemma 1}

Suppose that $t_{m}=t_{1}$. Then in regard to the truthfulness in revealing $\theta$, a pooling strategy cannot constitute the equilibrium.

\footnotetext{
${ }^{12}$ As mentioned in Okuno-Fujiwara, Postlewaite and Suzumura (1990), we believe that the condition that an agent reveals the private information truthfully should be an equilibrium conclusion rather than an assumption.
} 
Next M's separating strategies are " $a_{m}=h\left(a_{m}=l\right)$ if $\theta=h(\theta=l)$ " and " $a_{m}=h\left(a_{m}=l\right)$ if $\theta=l$ $(\theta=h)^{\prime}$. In the following, we show that the only separating strategy which constitutes an equilibrium is the first one, which implies that $\mathrm{M}$ is truthful in revealing $\theta$.

Without loss of generality, assume that $\theta=h$. Then, M's posterior beliefs regarding the true state are $\operatorname{Pr}(w=H \mid \theta=h)=p$ and $\operatorname{Pr}(w=L \mid \theta=h)=1-p$. In the following, $E \pi_{m}\left(a_{m}=h, \cdot, w\right)$ is M's expected payoff when $a_{m}=h$ for given $\theta=h$ (that is, when he is truthful in revealing $\theta$ ) and $E \pi_{m}\left(a_{m} \neq \theta, \cdot, w\right)$ is the expected payoff when $a_{m}=l$ for given $\theta=h$ (that is, when he deviates from $\theta$ ). If $t_{m}=t_{1}$ and therefore M's forecast is observed, U decides whether to imitate or deviate from $a_{m}$.

Consider the case in which $\mathrm{U}$ imitates $a_{m}$. Then,

$$
\begin{aligned}
E \pi_{m}\left(a_{m}=h, a_{u}=a_{m}, w \mid \theta=h\right) & =\sum_{w \in\{H, L\}} \operatorname{Pr}(w \mid \theta=h) \pi_{m}\left(a_{m}=h, a_{u}=a_{m}, w\right) \\
& =2 p-1 \\
E \pi_{m}\left(a_{m}=l, a_{u}=a_{m}, w \mid \theta=h\right) & =\sum_{w \in\{H, L\}} \operatorname{Pr}(w \mid \theta=h) \pi_{m}\left(a_{m} \neq h, a_{u}=a_{m}, w\right) \\
& =1-2 p
\end{aligned}
$$

Next, consider the case in which U deviates from $a_{m}$. Then,

$$
\begin{aligned}
E \pi_{m}\left(a_{m}=h, a_{u} \neq a_{m}, w \mid \theta=h\right) & =\sum_{w \in\{H, L\}} \operatorname{Pr}(w \mid \theta=h) \pi_{m}\left(a_{m}=h, a_{u} \neq a_{m}, w\right) \\
& =p \gamma-(1-p) \phi \\
E \pi_{m}\left(a_{m}=l, a_{u} \neq a_{m}, w \mid \theta=h\right) & =\sum_{w \in\{H, L\}} \operatorname{Pr}(w \mid \theta=h) \pi_{m}\left(a_{m}=l, a_{u} \neq a_{m}, w\right) \\
& =-p \phi+(1-p) \gamma
\end{aligned}
$$

Now, let $\mu \in[0,1]$ be the probability that $\mathrm{U}$ imitates M's action, i.e., $\mu=\operatorname{Pr}\left(a_{u}=a_{m}\right)$. Then,

$$
\begin{aligned}
& E \pi_{m}\left(a_{m}=h, a_{u}, w \mid \theta=h\right)-E \pi_{m}\left(a_{m}=l, a_{u}, w \mid \theta=h\right) \\
= & \mu\left(\sum_{w \in\{H, L\}} \operatorname{Pr}(w \mid h) \pi_{m}\left(a_{m}=h, a_{u}=a_{m}, w\right)\right)+(1-\mu)\left(\sum_{w \in\{H, L\}} \operatorname{Pr}(w \mid h) \pi_{m}\left(a_{m}=h, a_{u} \neq a_{m}, w\right)\right) \\
& -\mu\left(\sum_{w \in\{H, L\}} \operatorname{Pr}(w \mid h) \pi_{m}\left(a_{m}=l, a_{u}=a_{m}, w\right)\right)-(1-\mu)\left(\sum_{w \in\{H, L\}} \operatorname{Pr}(w \mid h) \pi_{m}\left(a_{m}=l, a_{u} \neq a_{m}, w\right)\right) \\
= & -(\gamma \mu-2 \mu-\phi-\gamma+\mu \phi)(2 p-1)>0
\end{aligned}
$$

because $\gamma \mu-2 \mu-\phi-\gamma+\mu \phi<0$ for all $\mu \in[0,1] .{ }^{13}$ Thus, regardless of U's best response in round 2, M's best response is to reveal his private information truthfully. Also note that this result holds for all $p \in\left(\frac{1}{2}, 1\right)$. In the following sections where $p$ is private information, $\mathrm{U}$ forms a posterior belief regarding $p$ from observing M's timing of forecast. For example, if his posterior belief regarding $p$ from observing

\footnotetext{
${ }^{13} \gamma \mu-2 \mu-\phi-\gamma+\mu \phi=\mu(\gamma+\phi-2)-\gamma-\phi$. So $\mu \gtreqless \frac{\gamma+\phi}{\gamma+\phi-2} \Longrightarrow \gamma \mu-2 \mu-\phi-\gamma+\mu \phi \gtreqless 0$. However as $\frac{\gamma+\phi}{\gamma+\phi-2}-1=$ $\frac{2}{\gamma+\phi-2}>0$, for all $\mu \in[0,1], \gamma \mu-2 \mu-\phi-\gamma+\mu \phi<0$.
} 
$t_{m}=t_{1}$ is $p \in(a, b)$ where $a, b \in\left[\frac{1}{2}, 1\right]$ and $a<b$, still it should be true that $a_{m}=\theta$ because the truthfulness should hold for all $p \in\left(\frac{1}{2}, 1\right)$. The case in which $\theta=l$ should yield the same result because both cases are ex-ante symmetric. This then implies that if $t_{m}=t_{1}$, the only equilibrium is the separating equilibrium in which $\mathrm{M}$ is truthful in revealing $\theta$.

\section{Lemma 2}

When $t_{m}=t_{1}$, the unique equilibrium is a separating equilibrium in which $M$ reveals $\theta$ truthfully, i.e., $a_{m}=\theta$.

Next, consider the case in which $\mathrm{M}$ decides to forecast in round 2. In this case, $\mathrm{M}$ knows that $\mathrm{U}$ randomizes his forecast because $\mathrm{U}$ has only the prior belief for the true state that $\operatorname{Pr}(w=H)=\operatorname{Pr}(w=$ $L)=0.5$. Although $\mathrm{U}$ forms a posterior belief for $p$ from observing $t_{m}=t_{2}$, as $a_{m}$ is not observable, no valuable information about the true state is conveyed. Here, we assume the following:

\section{Assumption 1}

Suppose U has no chance to observe $a_{m}$. In this case, $M$ believes that $\operatorname{Pr}\left(a_{u}=h\right)=\operatorname{Pr}\left(a_{u}=l\right)=0.5$.

Without loss of generality, assume that $\theta=h$. Then, under $\operatorname{Pr}(w=H \mid \theta=h)=p$ and $\operatorname{Pr}(w=L \mid \theta=$ $h)=1-p$,

$$
\begin{aligned}
E \pi_{m}\left(a_{m}=h, a_{u}, w \mid \theta=h\right) & =\frac{1}{2}\left(\sum_{a_{u} \in\{h, l\}}\left(\sum_{w \in\{H, L\}} \operatorname{Pr}(w \mid \theta) \pi_{m}\left(a_{m}=h, a_{u}, w\right)\right)\right) \\
& =\frac{1}{2}(2 p-\phi+p \gamma+p \phi-1) \\
E \pi_{m}\left(a_{m}=l, a_{u}, w \mid \theta=h\right) & =\frac{1}{2}\left(\sum_{a_{u} \in\{h, l\}}\left(\sum_{w \in\{H, L\}} \operatorname{Pr}(w \mid \theta) \pi_{m}\left(a_{m}=l, a_{u}, w\right)\right)\right) \\
& =\left(-\frac{1}{2}\right)(2 p-\gamma+p \gamma+p \phi-1)
\end{aligned}
$$

and

$$
E \pi_{m}\left(a_{m}=h, a_{u}, w \mid \theta=h\right)-E \pi_{m}\left(a_{m}=l, a_{u}, w \mid \theta=h\right)=\frac{1}{2}(2 p-1)(\gamma+\phi+2)>0
$$

Therefore, for all $p \in\left(\frac{1}{2}, 1\right), \gamma>1$ and $\phi>1$, if $t_{m}=t_{2}$, M's best response is to reveal $\theta$ truthfully. The above analysis can be summarized as follows:

\section{Proposition 1}

$M$ reveals her signal $\theta$ truthfully for all $p \in\left(\frac{1}{2}, 1\right)$ regardless of the timing of his forecast.

If we recall the given ex-post payoff structure (Table 1), the result that $M$ has no incentive to ignore his own information $\theta$ is quite intuitive. According to Table 1, the ex-post payoffs under $a_{i} \neq w$ are strictly smaller than those under $a_{i}=w$. That is, Min $\pi_{i}\left(a_{i}=w, a_{-i}\right)=1>-1=\operatorname{Max} \pi_{i}\left(a_{i} \neq w, a_{-i}\right)$. Therefore it is natural that M's primary objective should be to make a correct forecast. Hence, when no other valuable information is available, $\mathrm{M}$ has no incentive to ignore $\theta$, which is meaningful in the sense that it is correlated with the true state. This is why M's strategy to deviate from $\theta_{i}$ is strictly dominated. 
An important implication of Proposition 1 is that, especially when $t_{m}=t_{1}$, because of the requirement that U's belief should be consistent in equilibrium, $\mathrm{U}$ assigns zero probability to the possibility that $\mathrm{M}$ deviates from $\theta$ when $t_{m}=t_{1}$, i.e., $\lambda\left(\theta=a_{m}\right)=1$ where $\lambda(\cdot)$ is U's belief that M's is truthful in revealing $\theta$. That is, if $a_{m}$ is observable, $\mathrm{U}$ can infer $\theta$ perfectly. From M's standpoint of view, it also implies that the only option he can use to maximize his ex-ante expected payoff is a decision on the timing of forecast. Moreover, as he should reveal $\theta$ truthfully regardless of his timing of forecast, the decision on the timing of forecast is no more than a decision on the disclosure of his private signal $\theta$ : If $t_{m}=t_{1}$, it is in order to reveal $\theta$ to $\mathrm{U}$ and if $t_{m}=t_{2}$, it is in order to prevent $\theta$ from being revealed to $\mathrm{U}$.

\section{Equilibrium}

\subsection{Partially revealing separating equilibrium}

In our model, M's information quality, which is his type, is a continuum, i.e., $p \in\left(\frac{1}{2}, 1\right)$, while the action set is discrete, i.e., $t_{m} \in\left\{t_{1}, t_{2}\right\}$. Hence the observance of M's timing of forecast does not reveal M's type $p$ perfectly. It is in this sense that we use the term "partially revealing separating equilibrium".

In round $2, \mathrm{U}$ faces one of the following two situations: $t_{m}=t_{1}$ and $t_{m}=t_{2}$. As mentioned, if $\mathrm{M}$ did not forecast in round 1, i.e., $t_{m}=t_{2}$, U's best response in round 2 is to randomize his forecast, i.e., $z=[0,1]$ where $z=\operatorname{Pr}\left(a_{u}=h\right)$ because no information correlated with the true state is available and both $a_{u}=h$ and $a_{u}=l$ attain the same ex-ante expected payoffs.

Next, consider the case in which $\mathrm{M}$ forecasted in round 1, i.e., $t_{m}=t_{1}$. Note that originally $\mathrm{U}$ faces two dimensions of incomplete information; one regarding $\theta \in\{h, l\}$ and the other regarding $p \in\left(\frac{1}{2}, 1\right)$. However, from Proposition 1, U can infer $\theta$ perfectly from observing $a_{m}$. Hence, what we have to consider now is solely U's posterior belief regarding $p$ inferred from observing that $t_{m}=t_{1}$. That is, $t_{m}$ conveys information about $p$ and $\mathrm{U}$ infers it based on his belief.

Here, we assume that M's strategy as to the timing of forecast is a monotone function of the information quality. In the following, as we are considering a partially revealing separating equilibrium, $x, y \neq \frac{1}{2}$ and $x, y \neq 1$.

\section{Definition 2}

1) Monotone increasing strategy: $\exists x \in\left(\frac{1}{2}, 1\right)$ s.t. for $p \in\left(\frac{1}{2}, x\right), t_{m}=t_{1}$ and for $p \in(x, 1), t_{m}=t_{2}$.

2) Monotone decreasing strategy: $\exists y \in\left(\frac{1}{2}, 1\right)$ s.t. for $p \in(y, 1), t_{m}=t_{1}$ and for $p \in\left(\frac{1}{2}, y\right), t_{m}=t_{2}$.

Even $\mathrm{M}$ knows that if he forecasts in round 1, $\mathrm{U}$ can infer $\theta$ perfectly. As no cost is imposed for a delay of forecast, if $\mathrm{M}$ forecasts in round 1 it must be assumed that this is his voluntarily decision, unconstrained by considerations other than the usefulness of the delay. That is, if $\mathrm{M}$ forecasts without delaying until round 2 , it is in order to give $\mathrm{U}$ a chance to infer $\theta$ perfectly. Also, the monotone increasing (decreasing) strategy means that he wants to reveal information which is of relatively low (high) quality.

Below it is shown that the conditions under which the conjectured M's strategy is supported as the equilibrium strategy are described as a function of $\gamma$ and $\phi$. Therefore, the derived results show how the given payoff structure, especially the asymmetry in reward and penalty, affects M's decision on the timing of forecast and U's best response. In particular, as M's decision on the timing of forecast is no more than a decision on the disclosure of his superior information, the results provide the reasoning behind when and why $\mathrm{M}$ intends to reveal his valuable private information and allow $\mathrm{U}$ to infer it. 


\subsubsection{Monotone increasing strategy}

First, we assume that M's strategy is monotone increasing. Without loss of generality, assume that $a_{m}=h$. From Lemma 2, after observing $a_{m}=h$, U infers that $\theta=h$. Also, U has a posterior belief regarding $p$ such that $p \in\left(\frac{1}{2}, x\right)$. Then,

$$
\begin{aligned}
E \pi_{u}\left(a_{u}=a_{m}, w \mid t_{m}=t_{1}\right) & =\int\left(\sum_{w \in\{H, L\}} \operatorname{Pr}(w \mid \theta=h) \pi_{u}\left(a_{u}=a_{m}, w\right)\right) d Z\left(p \mid t_{m}=t_{1}\right) \\
& =\int_{p \in\left(\frac{1}{2}, x\right)}(p-(1-p)) d Z(p) \\
& =\frac{1}{2}(2 x-1) \\
E \pi_{u}\left(a_{u} \neq a_{m}, w \mid t_{m}=t_{1}\right) & =\int\left(\sum_{w \in\{H, L\}} \operatorname{Pr}(w \mid \theta=h) \pi_{u}\left(a_{u} \neq a_{m}, w\right)\right) d Z\left(p \mid t_{m}=t_{1}\right) \\
& =\int_{p \in\left(\frac{1}{2}, x\right)}(p(-\phi)+(1-p) \gamma) d Z(p) \\
& =\left(-\frac{1}{4}\right)(\phi-3 \gamma+2 x \gamma+2 x \phi)
\end{aligned}
$$

where (1) is the expected payoff when U imitates M's forecast and (2) is the expected payoff when U deviates from M's forecast. Then,

$$
E \pi_{u}\left(a_{u}=a_{m}, w \mid t_{m}=t_{1}\right)-E \pi_{u}\left(a_{u} \neq a_{m}, w \mid t_{m}=t_{1}\right)=\frac{1}{4}(4 x-3 \gamma+\phi+2 x \gamma+2 x \phi-2)
$$

We denote

$$
f(x) \equiv 4 x-3 \gamma+\phi+2 x \gamma+2 x \phi-2
$$

Then, U's best response in round 2 when $a_{m}$ is observable can be described as follows: i) $f(x)>0 \Longrightarrow$ $U$ imitates $a_{m}$, ii) $f(x)<0 \Longrightarrow U$ deviates from $a_{m}$, and iii) $f(x)=0 \Longrightarrow U$ is indifferent between imitating and deviating from $a_{m}$.

In the following, we denote $E \pi_{m}\left(t_{m}\right)$ as M's ex-ante expected payoff when he forecasts at $t_{m} \in\left\{t_{1}, t_{2}\right\}$. Note that if $t_{m}=t_{2}$, $\mathrm{U}$ randomizes his forecast in round 2 and in this case, from this assumption, $\mathrm{M}$ believes that $\operatorname{Pr}\left(a_{u}=h\right)=\operatorname{Pr}\left(a_{u}=l\right)=\frac{1}{2}$. Then,

$$
\begin{aligned}
E \pi_{m}\left(t_{m}=t_{2}\right) & =\frac{1}{2}\left(\sum_{a_{u} \in\{h, l\}}\left(\sum_{w \in\{H, L\}} \operatorname{Pr}(w \mid \theta=h) \pi_{m}\left(a_{m}, a_{u}, w\right)\right)\right) \\
& =\frac{1}{2}(2 p-\phi+p \gamma+p \phi-1)
\end{aligned}
$$

CASE 1: When $f(x)>0$.

In this case, if $\mathrm{M}$ forecasts in round 1, U imitates M's forecast in round 2. Then,

$$
\begin{aligned}
E \pi_{m}\left(t_{m}=t_{1}\right) & =\sum_{w \in\{H, L\}} \operatorname{Pr}(w \mid \theta=h) \pi_{m}\left(a_{m}, a_{u}=a_{m}, w\right) \\
& =2 p-1
\end{aligned}
$$


Hence from (3) and (4),

$$
E \pi_{m}\left(t_{m}=t_{1}\right)-E \pi_{m}\left(t_{m}=t_{2}\right)=\left(-\frac{1}{2}\right)(p \gamma-\phi-2 p+p \phi+1)
$$

The computation then yields the following result.

\section{Lemma 3}

Suppose that $\gamma<\phi$. If $p \in\left(\frac{1}{2}, \frac{\phi-1}{\gamma+\phi-2}\right), t_{m}=t_{1}$ and if $p \in\left(\frac{\phi-1}{\gamma+\phi-2}, 1\right), t_{m}=t_{2}$. If $t_{m}=t_{1}, U$ imitates $a_{m}$.

\section{Proof of Lemma 3}

In the appendix.

CASE 2: When $f(x)<0$.

In this case, if $\mathrm{M}$ forecasts in round $1, \mathrm{U}$ deviates from M's forecast in round 2. Then,

$$
\begin{aligned}
E \pi_{m}\left(t_{m}=t_{1}\right) & =\sum_{w \in\{H, L\}} \operatorname{Pr}(w \mid \theta) \pi_{m}\left(a_{m} \neq a_{u}\right) \\
& =p \gamma-\phi+p \phi
\end{aligned}
$$

From (3) and (6),

$$
E \pi_{m}\left(t_{m}=t_{1}\right)-E \pi_{m}\left(t_{m}=t_{2}\right)=p\left(\frac{1}{2} \gamma+\frac{1}{2} \phi-1\right)+\frac{1}{2}-\frac{1}{2} \phi
$$

Then, if $p \gtreqless \frac{\phi-1}{\gamma+\phi-2}, E \pi_{m}\left(t_{m}=t_{1}\right) \gtreqless E \pi_{m}\left(t_{m}=t_{2}\right)$. However, this is contradictory to the conjecture that M's strategy is monotone increasing. Hence, this case is excluded.

Case 3: When $f(x)=0$.

In this case, if $\mathrm{M}$ forecasts in round $1 \mathrm{U}$ is indifferent to imitating or deviating from M's forecast. Now, suppose that $z$ is the probability that U imitates $a_{m}$ when $a_{m}$ is observable, i.e., $z=\operatorname{Pr}\left(a_{u}=a_{m}\right)$. Then

$$
\begin{gathered}
E \pi_{m}\left(t_{m}=t_{1}\right)=z(p-(1-p))+(1-z)(p \gamma-(1-p) \phi) \\
E \pi_{m}\left(t_{m}=t_{2}\right)=\frac{1}{2}(p-(1-p))+\frac{1}{2}(p \gamma-(1-p) \phi)
\end{gathered}
$$

and

$$
E \pi_{m}\left(t_{m}=t_{1}\right)-E \pi_{m}\left(t_{m}=t_{2}\right)=\left(-\frac{1}{2}\right)(2 z-1)(p \gamma-\phi-2 p+p \phi+1)
$$

Hence if $z=\frac{1}{2}, E \pi_{m}\left(t_{m}=t_{1}\right)=E \pi_{m}\left(t_{m}=t_{2}\right)$ for all $p \in\left(\frac{1}{2}, 1\right)$. That is, when $a_{m}$ is observable, if $\mathrm{U}$ imitates (or deviates from) $a_{m}$ with probability $\frac{1}{2}, \mathrm{M}$ is indifferent regarding the choice between forecasting in round 1 or round 2 for all $p \in\left(\frac{1}{2}, 1\right)$. Now, a perfect Bayesian equilibrium requires that U's posterior belief over $p$ should be consistent to M's strategy. Thus, as $f(x)=0$ at $x=\frac{3 \gamma-\phi+2}{2 \gamma+2 \phi+4}$, we only have to check the condition under which $x=\frac{3 \gamma-\phi+2}{2 \gamma+2 \phi+4} \in\left(\frac{1}{2}, 1\right)$ is satisfied.

\section{Lemma 4}

Suppose that $\phi<\gamma<3 \phi+2$. Then, for $p \in\left(\frac{1}{2}, \frac{3 \gamma-\phi+2}{2 \gamma+2 \phi+4}\right), t_{m}=t_{1}$ and for $p \in\left(\frac{3 \gamma-\phi+2}{2 \gamma+2 \phi+4}, 1\right)$, $t_{m}=t_{2}$. If $t_{m}=t_{1}$, U imitates or deviates from $a_{m}$ with probability $\frac{1}{2}$.

\section{Proof of Lemma 4}

In the appendix. 


\subsubsection{Monotone decreasing strategy}

Now we assume that M's strategy is monotone decreasing. Without loss of generality, assume that $a_{m}=h$. After observing $a_{m}=h$, U infers that $\theta=h$. Also, U's posterior belief from observing $t_{m}=t_{1}$ is that $p \in(y, 1)$. Then

$$
\begin{aligned}
E \pi_{u}\left(a_{u}=a_{m}, w \mid t_{m}=t_{1}\right) & =\int\left(\sum_{w \in\{H, L\}} \operatorname{Pr}(w \mid \theta=h) \pi_{u}\left(a_{u}=a_{m}, w\right)\right) d Z\left(p \mid t_{m}=t_{1}\right) \\
& =\int_{p \in(y, 1)}(p-(1-p)) d Z(p) \\
& =y \\
E \pi_{u}\left(a_{u} \neq a_{m}, w \mid t_{m}=t_{1}\right) & =\int\left(\sum_{w \in\{H, L\}} \operatorname{Pr}(w \mid \theta=h) \pi_{u}\left(a_{u} \neq a_{m}, w\right)\right) d Z\left(p \mid t_{m}=t_{1}\right) \\
& =\int_{p \in(y, 1)}(p(-\phi)+(1-p) \gamma) d Z(p) \\
& =\left(-\frac{1}{2}\right)(\phi-\gamma+y \gamma+y \phi)
\end{aligned}
$$

where (11) is the expected payoff when U imitates M's action and (12) is the expected payoff when U deviates from M's action. Then,

$$
E \pi_{u}\left(a_{u}=a_{m}, w\right)-E \pi_{u}\left(a_{u} \neq a_{m}, w\right)=\frac{1}{2}(2 y-\gamma+\phi+y \gamma+y \phi)
$$

We denote

$$
g(y) \equiv 2 y-\gamma+\phi+y \gamma+y \phi
$$

U's best response in round 2 can thus be described as follows: i) $g(y)>0 \Longrightarrow U$ imitates $a_{m}$, ii) $g(y)<0 \Longrightarrow U$ deviates from $a_{m}$. iii) $g(y)=0 \Longrightarrow U$ is indifferent to the distinction between imitating and deviating from $a_{m}$. Then, from the procedure analogous to that used in the case in which M's strategy is monotone increasing, we can derive the following result.

\section{Lemma 5}

Suppose that $\gamma>3 \phi+2$. Then, for $p \in\left(\frac{\gamma-\phi}{\gamma+\phi+2}, 1\right), t_{m}=t_{1}$ and for $p \in\left(\frac{1}{2}, \frac{\gamma-\phi}{\gamma+\phi+2}\right), t_{m}=t_{2}$. If $t_{m}=t_{1}$, U imitates or deviates from $a_{m}$ with probability $\frac{1}{2}$.

\section{Proof of Lemma 5}

In the appendix.

Then, the results of the above analysis can be summarized as follows.

\section{Proposition 2: (Partially revealing) Separating equilibrium}

1) Suppose that $\gamma<\phi$. If $p \in\left(\frac{1}{2}, \frac{\phi-1}{\gamma+\phi-2}\right), t_{m}=t_{1}$ and if $p \in\left(\frac{\phi-1}{\gamma+\phi-2}, 1\right), t_{m}=t_{2}$. If $t_{m}=t_{1}, U$ imitates $a_{m}$.

2) Suppose that $\phi<\gamma<3 \phi+2$. If $p \in\left(\frac{1}{2}, \frac{3 \gamma-\phi+2}{2 \gamma+2 \phi+4}\right), t_{m}=t_{1}$ and if $p \in\left(\frac{3 \gamma-\phi+2}{2 \gamma+2 \phi+4}, 1\right), t_{m}=t_{2}$. If $t_{m}=t_{1}$, U imitates or deviates from $a_{m}$ with probability $\frac{1}{2}$. 
3) Suppose that $\gamma>3 \phi+2$. If $p \in\left(\frac{1}{2}, \frac{\gamma-\phi}{\gamma+\phi+2}\right), t_{m}=t_{2}$ and if $p \in\left(\frac{\gamma-\phi}{\gamma+\phi+2}, 1\right), t_{m}=t_{1}$. If $t_{m}=t_{1}$, $U$ imitates or deviates from $a_{m}$ with probability $\frac{1}{2}$.

For all three cases, if $t_{m}=t_{2}, U$ randomizes his forecast, i.e., $\operatorname{Pr}\left(a_{u}=h\right)=[0,1]$.

Consider the situation in which the payoff structure is biased toward the penalty, i.e., $\gamma<\phi$. If $\mathrm{M}$ forecasts without a delay, U infers that M's private signal $\theta$ is of low quality. Hence, the credibility he assigns to the correctness of M's information may not be high. However, as the payoff structure is biased toward the penalty, U's main concern is to avoid being the only agent who announces a wrong forecast. Hence, in spite of his weak belief for the correctness of $\theta$, U imitates M's forecast. Of course, as $\theta$ is correlated with the true state, it seems reasonable for $U$ to rely on it. However, it should be noted that the payoff structure biased toward the penalty also affects U's strategic behavior of imitating $a_{m}$. By forecasting identically, $\mathrm{U}$ can at least avoid the worst case in which he earns the lowest payoff $\pi_{u}=-\phi$ when the penalty is strongly stressed. U's response when the payoff structure is biased toward the reward, as stated below, clearly shows how the given payoff structure affects U's strategic response against the observed $a_{m}$ and the inferred $\theta$.

Although M observes an informative signal, even he is not free from the concern that he could be penalized because his information is not perfect. However, at least as $\mathrm{M}$ observes the signal correlated with true state, his belief for the correctness of his information matters. If it is relatively high, i.e., $p \in$ $\left(\frac{\phi-1}{\gamma+\phi-2}, 1\right), \mathrm{M}$ gives more weight to the possibility that his information may be correct. If forecast turns out to be correct, U's identical forecast causes a negative payoff externality because a good reputation or reward must be shared. Thus, he regards U's identical forecast as a strategic substitute and therefore wants to prevent his private signal from being revealed. This is why $\mathrm{M}$ delays his forecast when his information is of relatively high quality. On the other hand, if the precision of his private signal is relatively low, i.e., $p \in\left(\frac{1}{2}, \frac{\phi-1}{\gamma+\phi-2}\right)$, M is not quite sure about the correctness of his information, so he is greatly concerned about the possibility that he could be penalized alone. If his forecast turned out to be wrong, the other's identical forecast would cause a positive payoff externality because the penalty or blame would be shared. Hence, in this case, M regards U's identical forecast as a strategic complement and therefore wants to induce it by revealing his private signal to U. This explains why $M$ forecasts without a delay when his information is of low quality, even when a waiting option is not costly.

Next, consider the case in which the payoff structure is biased toward the reward, i.e., $\gamma>\phi$. Note that the reward $\gamma$ can be earned only if an agent is the only one who forecasted correctly. That is, alongside being correct, forecasting differently from the other player is a necessary condition for earning $\gamma$. So the incentive to be differentiated is initiated. However, how strongly the reward is stressed over the penalty matters because a different forecast bears a risk of announcing a wrong forecast alone. That is, each player considers whether the reward is sufficiently large enough to make him risk of being penalized alone.

Suppose that $\phi<\gamma<3 \phi+2$. This corresponds to the case in which the payoff structure is weakly biased toward the reward. If $\mathrm{M}$ forecasts without a delay, $\mathrm{U}$ infers that $\theta$ is of low quality. However, $\mathrm{U}$ also intends to be differentiated. Hence, compared to the case in which the penalty is stressed, U has a relatively small incentive to imitate M's forecast when it is observable. This may lead U to use a mixed strategy and sometimes deviate from M's forecast in order to earn $\gamma$. Actually, if U uses a mixed strategy, $\mathrm{M}$ is indifferent between $t_{m}=t_{1}$ and $t_{m}=t_{2}$ for all $p \in\left(\frac{1}{2}, 1\right)$. The condition that U's belief over $p$ should be consistent to M's equilibrium strategy yields that M reveals his private signal only if it is of low 
quality, i.e., $p \in\left(\frac{1}{2}, \frac{3 \gamma-\phi+2}{2 \gamma+2 \phi+4}\right)$. Although the payoff structure is biased toward the reward, the reward is not sufficiently large enough to make him take the risk of being penalized alone. Hence, like the case in which the penalty is stressed, he reveals his information if it is of low quality, in order to be imitated by U.

On the other hand, if $\gamma>3 \phi+2$, this corresponds to the case in which the payoff structure is strongly biased toward the reward. In an equilibrium, U infers that M's revealed information is of high quality. However, he uses a mixed strategy and deviates from M's forecast with a positive probability while ignoring $a_{m}$ based on the high quality information. Interestingly, M's information is revealed only if it is of high quality, i.e., $t_{m}=t_{1}$ for $p \in\left(\frac{\gamma-\phi}{\gamma+\phi+2}, 1\right)$. This equilibrium strategy for $\mathrm{M}$ can be explained by the following reasoning. When $\mathrm{M}$ expects that $\mathrm{U}$ is likely to deviate from $a_{m}$, if he intends to reveal his private signal it is better to do so when the precision of his signal is relatively high. If $U$ deviates from what the relatively imprecise information signals, U's forecast is more likely to be correct compared to the case in which $U$ deviates from what the relatively precise information signals. That is, if information is relatively precise, it is better to reveal it. On the other hand, if information is relatively imprecise, it is better to conceal it. Therefore, $\mathrm{M}$ reveals his information only if it is of high quality. In this way, the payoff structure which is strongly biased toward the reward induces both players to take the risk of being penalized alone, which yields the interesting result that M's private signal is revealed only if it is of high quality.

In sum, there exists a partially revealing separating equilibrium in which $\mathrm{M}$ forecasts voluntarily without a delay in order to reveal his private signal to U. However, M's motivations for acting so differ according to the given payoff structures. If the penalty is larger than the reward or the reward is weakly larger than the penalty, M's information is revealed only if it is of low quality, in order to induce U's imitation for the sake of avoiding the risk of being penalized alone. On the other hand, if the reward is sufficiently larger than the penalty, M's information is revealed only if it is of high quality, in order to induce U's divergence for the sake of earning a reward alone. In particular, the result that, according to whether $\phi<\gamma<3 \phi+2$ or $\gamma>3 \phi+2$, the different quality of information is revealed against U's same equilibrium strategy also reveals how the given payoff structure affects M's strategic decision on the disclosure of his valuable information.

\subsection{Pooling equilibrium}

According to the set-up of the model, $\mathrm{M}$ has two pooling strategies: i) $t_{m}=t_{1}$ for all $p \in\left(\frac{1}{2}, 1\right)$ and ii) $t_{m}=t_{1}$ for all $p \in\left(\frac{1}{2}, 1\right)$. As we are primarily interested in M's strategic incentive to reveal his information, in this section we restrict our attention to the pooling equilibrium in which $\mathrm{M}$ forecasts without a delay for all qualities of information. ${ }^{14}$

Consider the following strategy for $\mathrm{M}: t_{m}=t_{1}$ for all $p \in\left(\frac{1}{2}, 1\right)$. If $\mathrm{U}$ observes that $t_{m}=t_{1}$ which is on the equilibrium path, U's posterior belief regarding $p$ is $p \in\left(\frac{1}{2}, 1\right)$. On the other hand, for the situation in which $\mathrm{U}$ observes that $t_{m}=t_{2}$ which never occurs along the equilibrium path, we can assign any value to U's arbitrary posterior belief regarding $p$. However, it is important to note that, regardless of U's posterior belief regarding $p$, U's best response in round 2 is still to randomize his forecast because $a_{m}$ is not observable and therefore $\theta$ cannot be inferred. That is, U's posterior belief regarding $p$ from observing $t_{m}=t_{2}$, which is off-the-equilibrium path, does not affect U's best response and therefore it

\footnotetext{
${ }^{14}$ The other pooling equilibrium in which $t_{m}=t_{2}$ for all $p \in\left(\frac{1}{2}, 1\right)$ is discussed in Section 6.3.
} 
does not affect M's decision on $t_{m} \in\left\{t_{1}, t_{2}\right\}$. What $\mathrm{M}$ should consider is whether he intends to i) show $\theta$ in order to induce U's imitation or deviation or ii) conceal $\theta$ and make $\mathrm{U}$ randomize a forecast.

The analysis yields the following result:

\section{Proposition 3: Pooling equilibrium}

1) Suppose that $\gamma>3 \phi+2$. Then, $t_{m}=t_{1}$ for all $p \in\left(\frac{1}{2}, 1\right)$. If $t_{m}=t_{1}$, $U$ deviates from $a_{m}$.

2) Suppose that $\gamma=3 \phi+2$. Then, $t_{m}=t_{1}$ for all $p \in\left(\frac{1}{2}, 1\right)$. If $t_{m}=t_{1}$, $z \leq \frac{1}{2}$ where $z=$ $\operatorname{Pr}\left(a_{u}=a_{m}\right)$.

For both cases, if $t_{m}=t_{2}$, U randomizes his forecast in round 2.

\section{Proof of Proposition 3}

In the appendix.

Proposition 3 indicates that for the pooling equilibrium in which $t_{m}=t_{1}$ for all $p \in\left(\frac{1}{2}, 1\right)$ to be supported, i) the reward should be sufficiently larger than the penalty and ii) U deviates from $a_{m}$ when observable. If the reward is strongly stressed, even $\mathrm{U}$, who is uninformed about the true state, intends to deviate from $a_{m}$ if observable because he is willing to take the risk of being penalized alone for the sake of earning $\gamma$. This incentive to be differentiated also applies to M. If $\mathrm{M}$ expects that $\mathrm{U}$ intends to be differentiated always by deviating from $a_{m}$, then it would be better for $\mathrm{M}$ to always reveal his signal to U. If he delays his forecast and therefore $\mathrm{U}$ has no chance to observe $a_{m}$, it sometimes may be that $a_{m}=a_{u}$ because $\mathrm{U}$ randomizes a forecast. On the other hand, if he forecasts without a delay and therefore $a_{m}$ is revealed, one necessary condition for earning $\gamma$, which is that $a_{m} \neq a_{u}$, is always guaranteed. This explains why $\mathrm{M}$ always reveals his private signal to $\mathrm{U}$.

\section{Discussion}

\subsection{When information quality is public information}

In this section, in the interest of comparison, we consider the case in which the precision of M's private signal is public information. Note that Proposition 1, which states that M's best response is to reveal $\theta$ truthfully, should also hold in this case because being truthful is his best response for all $p \in\left(\frac{1}{2}, 1\right)$.

First, we derive U's best response in round 2. If $t_{m}=t_{2}$, U's best response is to randomize his forecast. If $t_{m}=t_{1}$, U observes $a_{m}$ and infers $\theta$ perfectly. In this case, U' best response in round 2 is as follows:

\section{Lemma 6}

Suppose that $p$ is public information. When $t_{m}=t_{1}$, U's best response in round 2 can be described as follows:

1) if $\gamma>\phi$, for $p \in\left(\frac{1}{2}, \frac{\gamma+1}{\gamma+\phi+2}\right)$, $U$ deviates from $a_{m}$ and for $p \in\left(\frac{\gamma+1}{\gamma+\phi+2}, 1\right), U$ imitates $a_{m}$.

2) if $\gamma \leq \phi$, $U$ imitates $a_{m}$ for all $p \in\left(\frac{1}{2}, 1\right)$.

\section{Proof of Lemma 6}

In the appendix.

Now using backward induction, we derive M's decision on the timing of forecast. 


\section{Proposition 4}

Suppose that $p$ is public information. Then, M's strategic decision on the timing of forecast can be described as follows:

1) Suppose $\gamma<\phi$. If $p \in\left(\frac{1}{2}, \frac{\phi-1}{\gamma+\phi-2}\right), t_{m}=t_{1}$ and if $p \in\left(\frac{\phi-1}{\gamma+\phi-2}, 1\right), t_{m}=t_{2}$.

2) Suppose $\gamma>\phi$. If $p \in\left(\frac{1}{2}, \frac{\gamma+1}{\gamma+\phi+2}\right), t_{m}=t_{1}$ and if $p \in\left(\frac{\gamma+1}{\gamma+\phi+2}, 1\right), t_{m}=t_{2}$.

3) Suppose $\gamma=\phi$. Then, for all $p \in\left(\frac{1}{2}, 1\right), t_{m}=t_{2}$.

\section{Proof of Proposition 4}

In the appendix.

Consider the situation in which the reward and the penalty are asymmetric. Contrary to the case in which $p$ is private information, if $a_{m}$ is observable, $\mathrm{U}$ decides his best response while being perfectly informed about the precision of M's signal. The most distinctive result is derived when the payoff structure is biased toward the reward because, unlike the case where $p$ is private information, $\mathrm{M}$ always intend to be differentiated from $\mathrm{U}$ in order to earn $\gamma$. When $a_{m}$ is observable and therefore $\theta$ is inferable, although $\mathrm{U}$ knows that it is correlated with true state, if the precision is relatively low he does not give much credit to the correctness of M's information, i.e., $p \in\left(\frac{1}{2}, \frac{\gamma+1}{\gamma+\phi+2}\right)$. Therefore, U diverges from it. If not, i.e., $p \in\left(\frac{\gamma+1}{\gamma+\phi+2}, 1\right)$, although $\mathrm{U}$ still has an incentive to be differentiated, he considers the fact that making a correct forecast is necessary to avoid a penalty. Then, as it is not quite attractive to ignore very precise information, the incentive to be differentiated is dominated and U imitates M's forecast. As the reward is stressed, $\mathrm{M}$ also wants to be differentiated by inducing both agents' different forecasts. He knows that, when $p$ is relatively high, if $\theta$ is revealed, $\mathrm{U}$ imitates it and the opportunity for him to earn $\gamma$ is foregone. Hence, he uses a waiting option to prevent his private signal from being revealed. On the other hand, if $p$ is relatively low, i.e., $p \in\left(\frac{1}{2}, \frac{\gamma+1}{\gamma+\phi+2}\right)$, M knows that $\mathrm{U}$ will deviate form his forecast if it is observable. In this situation, it would be better for $\mathrm{M}$ to reveal $\theta$ in order to guarantee that $a_{m} \neq a_{u}$, which is a necessary condition for earning $\gamma$. Here we can think about the possibility that the private signal of low quality is revealed in order to be imitated, like the case in which $p$ is private information. However note that M's strategic disclosure of low quality information is made while knowing that $U$ will deviate from $a_{m}$ if observable. Also it can be verified that $\frac{\partial\left(\frac{\gamma+1}{\gamma+\phi+2}\right)}{\partial \gamma}>0$ and $\frac{\partial\left(\frac{\gamma+1}{\gamma+\phi+2}\right)}{\partial \phi}<0$, which imply that as the reward is more heavily stressed than the penalty, the parameter set of $p$ for which M reveals his signal increases. This result implicitly supports the reasoning that M's disclosure of low quality information is based on the incentive to induce U's divergence. If the payoff structure is biased toward the penalty, the same equilibrium as in the case in which $p$ is private information is derived, from the same reasoning.

In sum, if $p$ is public information, M's private signal is revealed only if it is of low quality. However, the reasoning through which its disclosure is derived are different according to the given payoff structure. If the penalty is stressed, the disclosure is motivated by the desire to be imitated for the sake of avoiding the risk of being penalized alone. This is supported by U's equilibrium strategy, which is to imitate M's forecast whenever it is observable. On the other hand, if the reward is stressed, the information disclosure is an attempt to induce U's divergence for the sake of earning the reward alone. This is supported by U's equilibrium strategy, which is to deviate from M's forecast when the precision of information is relatively low. If M's information is of high quality, it is not revealed at all because of M's incentive to conceal his valuable information which is of high quality for the sake of avoiding U's identical forecast. 


\section{Corollary 1}

If $p$ is public information, there exists no equilibrium in which M's very precise information is revealed. That is, incomplete information about $p$ is a necessary condition for the strategic disclosure of M's very precise information.

\subsection{When the reward and the penalty are symmetric}

In previous sections, we have considered the case in which the reward and the penalty are asymmetric. According to Proposition 2 and Proposition 3, both the partially revealing separating equilibrium and the pooling equilibrium in which $t_{m}=t_{1}$ for all $p \in\left(\frac{1}{2}, 1\right)$ are not supported when the reward and the penalty are symmetric. This implicitly implies that if the reward and the penalty are symmetric, the only equilibrium is the pooling equilibrium in which M's signal is not revealed for all $p \in\left(\frac{1}{2}, 1\right)$.

Consider the following pooling strategy for $\mathrm{M}$ : " $t_{m}=t_{2}$ for all $p \in\left(\frac{1}{2}, 1\right)$ ". If U observes that $\mathrm{M}$ did not forecast in round 1, although U's posterior belief regarding $p$ is $p \in\left(\frac{1}{2}, 1\right)$, it does not affect the fact that $\mathrm{U}$ should randomize his forecast in round 2 because $a_{m}$ is not observable. On the other hand, if $\mathrm{U}$ observes that $t_{m}=t_{1}$ which never occurs along the equilibrium path, any arbitrary posterior belief over $p$ can be assigned to $\mathrm{U}$. In the following, we show that if $\gamma=\phi$, the given pooling strategy is supported as the equilibrium strategy whether $U$ believes that M's strategy is monotone increasing or monotone decreasing.

First, consider the case in which U believes that M's strategy is monotone increasing; That is, $\exists x \in$ $\left(\frac{1}{2}, 1\right]$ such that If $p \in\left(\frac{1}{2}, x\right), t_{m}=t_{1}$ and if $p \in(x, 1), t_{m}=t_{2}$. Here, as we consider the case in which $\mathrm{U}$ observes that $t_{m}=t_{1}$, the case in which $x=\frac{1}{2}$ can be excluded. Then for $t_{m}=t_{1}$ which is off-the-equilibrium path, U's posterior belief regarding $p$ is $p \in\left(\frac{1}{2}, x\right)$ where $x \in\left(\frac{1}{2}, 1\right]$. If U's belief over $p$ is formed in this way, from (1) and (2),

$$
\begin{aligned}
E \pi_{u}\left(a_{u}=a_{m}, w \mid t_{m}=t_{1}\right) & =\frac{1}{2}(2 x-1)>0 \\
\left.E \pi_{u}\left(a_{u} \neq a_{m}, w \mid t_{m}=t_{1}\right)\right|_{\phi=\gamma} & =\left(-\frac{1}{2}\right) \gamma(2 x-1)<0
\end{aligned}
$$

So U's best response for $t_{m}=t_{1}$, which is off-the-equilibrium path, is always to imitate $a_{m}$ if it is observable.

Second, consider the situation in which U believes that M's strategy is monotone decreasing; that is, $\exists y \in\left[\frac{1}{2}, 1\right)$ such that If $p \in(y, 1), t_{m}=t_{1}$ and if $p \in\left(\frac{1}{2}, y\right), t_{m}=t_{2}$. As we consider the case in which $\mathrm{U}$ observes that $t_{m}=t_{1}$, the case in which $y=1$ is excluded. Then for $t_{m}=t_{1}$ which never occurs along the equilibrium path, U's posterior belief over $p$ is $p \in(y, 1)$ where $y \in\left[\frac{1}{2}, 1\right)$. If U's belief over $p$ is formed in this way, from (11) and (12),

$$
\begin{aligned}
E \pi_{u}\left(a_{u}=a_{m}, w \mid t_{m}=t_{1}\right) & =y>0 \\
\left.E \pi_{u}\left(a_{u} \neq a_{m}, w \mid t_{m}=t_{1}\right)\right|_{\phi=\gamma} & =-\gamma y<0
\end{aligned}
$$

So U's best response for $t_{m}=t_{1}$, which is off-the-equilibrium path, is always to imitate $a_{m}$ if it is observable.

Therefore, regardless of U's posterior belief, if $\gamma=\phi$, U's best response is to imitate $a_{m}$ if it is observable. Then if $\mathrm{U}$ deviates from the given pooling strategy and forecasts in round 1 , as $\mathrm{U}$ imitates $a_{m}$ always, from (4), M's expected payoff from deviating the pooling strategy is $E \pi_{m}\left(t_{m}=t_{1}\right)=2 p-1$. On 
the other hand, if he follows the given pooling strategy, from $(3),\left.E \pi_{m}\left(t_{m}=t_{2}\right)\right|_{\phi=\gamma}=\frac{1}{2}(2 p-1)(\gamma+1)$. Then

$$
E \pi_{m}\left(t_{m}=t_{1}\right)-\left.E \pi_{m}\left(t_{m}=t_{2}\right)\right|_{\phi=\gamma}=\left(-\frac{1}{2}\right)(2 p-1)(\gamma-1)<0
$$

So $\mathrm{M}$ has no incentive to deviate from the pooling strategy, which is $t_{m}=t_{2}$ for all $p \in\left(\frac{1}{2}, 1\right)$.

\section{Proposition 5}

Suppose that $\gamma=\phi$. Then the unique equilibrium is the pooling equilibrium in which $t_{m}=t_{2}$ for all $p \in\left(\frac{1}{2}, 1\right)$.

In addition, recall Proposition 4 which says that even in the case where $p$ is public information, if $\gamma=\phi, \mathrm{M}$ always forecasts in round 2 after a delay. Hence, it is verifiable that if the reward and the penalty are symmetric, M's private signal is never revealed whether $p$ is private or public information.

\section{Corollary 2}

Whether the quality of information is incomplete or not, the asymmetry in reward and penalty is the necessary condition for M's strategic disclosure of information.

\subsection{When is information not revealed under the asymmetry in reward and penalty?}

In previous sections, to focus on the matter of strategic information revelation within a competitive environment, we dealt only with the pooling equilibrium in which the informed agent forecasts without a delay. However, we can also consider the possibility that the informed agent delays his forecast always and therefore his meaningful information is not revealed at all.

Suppose that M's pooling strategy is given as follows: " $t_{m}=t_{2}$ for all $p \in\left(\frac{1}{2}, 1\right)$ ". If U observes that $t_{m}=t_{2}$ which is on the equilibrium path, U's best response in round 2 is still to randomize his forecast. On the other hand, if $\mathrm{U}$ observes that $t_{m}=t_{1}$ which is off-the-equilibrium path, $\mathrm{U}$ can assign an arbitrary posterior belief over $p$. Here, we still assume that M's strategy is monotone. If $\mathrm{M}$ forecasts in round 2 , it is in order to conceal his private signal. Then, M's pooling strategy " $t_{m}=t_{2}$ for all $p \in\left(\frac{1}{2}, 1\right)$ " is more likely to be sustained if $\mathrm{U}$ is more likely to imitate $a_{m}$ when it is observable. That is, between the beliefs that M's strategy is monotone increasing and monotone decreasing, the latter makes it harder for M to deviate from the given pooling strategy than the former because $\mathrm{U}$ is more likely to imitate $a_{m}$ when he believes that $a_{m}$ is based on the more precise information. Hence, we proceed with the analysis under the conjecture that M's strategy is monotone decreasing. Then, the analysis yields the following result:

\section{Proposition 6}

Suppose that $U$ believes that $M$ 's strategy is monotone decreasing for $t_{m}=t_{1}$ which is off-theequilibrium path. Then the pooling equilibrium in which $t_{m}=t_{2}$ for all $p \in\left(\frac{1}{2}, 1\right)$ is derived for following cases:

1) When $\phi<\gamma<3 \phi+2$, if $t_{m}=t_{1}$, U believes that $p \in(y, 1)$ where $y \in\left[\frac{1}{2}, 1\right)$ and imitates $a_{m}$.

2) When $\gamma=3 \phi+2$, if $t_{m}=t_{1}$, U believes that $p \in(y, 1)$ where $y \in\left(\frac{1}{2}, 1\right)$ and imitates $a_{m}$. If $y=\frac{1}{2}$, so $U$ is indifferent between imitating and deviating from $a_{m}$, it should be that $z>\frac{1}{2}$ where $z=\operatorname{Pr}\left(a_{u}=a_{m}\right)$. 
3) When $\gamma>3 \phi+2$, if $t_{m}=t_{1}$, U believes that $p \in(y, 1)$ where $y \in\left(\frac{\gamma-\phi}{\gamma+\phi+2}, 1\right)$ and imitates $a_{m}$. If $y=\frac{\gamma-\phi}{\gamma+\phi+2}$, so $U$ is indifferent between imitating and deviating from $a_{m}$, it should be that $z>\frac{1}{2}$ where $z=\operatorname{Pr}\left(a_{u}=a_{m}\right)$.

\section{Proof of Proposition 6}

In the appendix.

The common features of the conditions under which this pooling equilibrium can be sustained are as follows: i) the reward is greater than the penalty, ii) if $t_{m}=t_{1}$ which is the off-the-equilibriumpath, U forms a belief over $p$ under which he imitates $a_{m}$. The intuition for these two conditions is straightforward; if the reward is greater than the penalty, M's incentive to be differentiated is initiated. Hence, if $\mathrm{M}$ expects that $\mathrm{U}$ will imitate his forecast and therefore a necessary condition for earning $\gamma$ will not be satisfied, $\mathrm{M}$ does not want to reveal his information and therefore intends to conceal his private signal through a delay.

In addition, if we consider the case in which $U$ believes that M's strategy is monotone increasing for $t_{m}=t_{1}$ which is off-the-equilibrium path, it can be checked that the pooling equilibrium in which M's signal is not revealed can be sustained in following cases: a) When $\phi<\gamma<3 \phi+2$, if $x \in\left(\frac{(3 \gamma-\phi+2)}{2(\gamma+\phi+2)}, 1\right)$ or if $x=\frac{(3 \gamma-\phi+2)}{2(\gamma+\phi+2)}$ and $z \geq \frac{1}{2}$. c) When $\gamma=3 \phi+2$, if $z \geq \frac{1}{2}$. Hence, it is checked that the results derived in Proposition 6 cover these results.

\subsection{Evaluation of the equilibria in terms of expected payoff}

The derived results of the former sections can be summarized as follows:

\begin{tabular}{|l|l|l|l|l|l|}
\hline & $\gamma<\phi$ & $\gamma=\phi$ & $\phi<\gamma<3 \phi+2$ & $\gamma=3 \phi+2$ & $\gamma>3 \phi+2$ \\
\hline Partial revelation & $\sqrt{ }($ low $p)$ & & $\sqrt{ }^{*}($ low $p)$ & & $\sqrt{ }($ high $p)$ \\
\hline Full revelation & & & & $\sqrt{ }^{*}$ & $\sqrt{ }^{*}$ \\
\hline No revelation & & $\sqrt{ }$ & $\sqrt{ }$ & $\sqrt{ }$ & $\sqrt{ }$ \\
\hline
\end{tabular}

<Table 2: M's information revelation according to payoff structure>

If we focus on the equilibrium in which M's private signal is revealed (partial and full revelation equilibrium), we can see that the asymmetry in reward and penalty plays a critical role in deciding which quality of information is revealed. This reflects the situations in which agents hope for the best or prepare for the worst. In other words, if the stick is stressed, the agents are biased toward preparing for the worst case in which they may be penalized alone. If the carrot is strongly stressed, each agent is biased toward hoping for the best case in which he earns the reward alone.

Although we extend our interest into the equilibrium in which M's private signal is not revealed, if $\gamma \leq \phi$, there exists a unique equilibrium for each case. On the other hand, if $\gamma>\phi$, a multiplicity of the equilibrium is derived because, for the pooling equilibrium in which $t_{m}=t_{2}$ for all $p \in\left(\frac{1}{2}, 1\right)$, any arbitrary posterior belief over $p$ can be assigned to $\mathrm{U}$ for $t_{m}=t_{1}$ which is off-the-equilibrium path. Below, we evaluate the multiple equilibria of each case in terms of the expected payoff to see the relation of the 
equilibrium dominance. In table 2 , the equilibrium marked as $\sqrt{ } *$ denotes the dominant equilibrium in terms of expected payoff.

\section{Corollary 3}

The evaluation of the equilibria in terms of expected payoff yields the following results:

1) If $\gamma \geq 3 \phi+2$, "Full revelation equilibrium" is a dominant equilibrium.

2) If $\phi<\gamma<3 \phi+2$, "Partial revelation equilibrium" is a dominant equilibrium.

\section{Proof of Corollary 3}

In the appendix.

Therefore when the reward is greater than the penalty, under all cases, the pooling equilibrium in which M's private signal is not revealed for all $p$ is dominated in terms of expected payoff by other equilibrium in which strategic information disclosure is derived. ${ }^{15}$

\section{Concluding remarks}

In this article, we explore the informed agent's strategic incentive to reveal his valuable information within a competitive environment characterized by a relative evaluation scheme. To restrict our attention on the matter of the strategic disclosure of meaningful information, we allow a waiting option only to the informed player. As we also assume that a delay is not costly, the informed agent's decision on the timing of forecast is no more than the voluntary decision regarding the disclosure of his information. We show that, when the precision of the informed agent's information is private information, the asymmetry in the reward and penalty of the payoff structure plays an important role in the informed agent's decision regarding which quality of information will be revealed for which incentive.

If the penalty is larger than the reward or the reward is weakly larger than the penalty, his information is revealed only if it is of low quality; this revelation reflects the informed agent's desire to be imitated by the other agent in order to avoid the worst scenario, in which he alone fails and incurs a penalty. On the other hand, if the reward is sufficiently larger than the penalty, his information is revealed even though - or only if - it is of high quality, in order to induce the other's divergence for the sake of earning the reward alone. If we consider the case in which the precision of the informed agent's information is completely known, the informed agent's information is revealed only if it is of low quality. Hence, it is verified that incomplete information about the quality of the informed player's information is a necessary condition for the disclosure of his very precise information. Also, if the reward and the penalty are symmetric, the unique equilibrium is the one in which the informed agent's information is not revealed. Hence, the asymmetry in reward and penalty is a necessary condition for the disclosure of his meaningful information. If the reward is strictly greater than the penalty, there also exists pooling equilibrium in which the informed agent's information is not revealed at all. However, the evaluation of the equilibria in terms of expected payoff yields that such a no revelation equilibrium is dominated by the partial- or full revelation equilibrium for all cases.

\footnotetext{
${ }^{15}$ As is well known, the intuitive criterion D1 and D2 are usually used to eliminate the equilibrium based on the irrational belief in the off-the-equilibrium path. However, in this model, the informed agent's type, $p \in\left(\frac{1}{2}, 1\right)$, is a continuum and the action set, $t_{m}=\left\{t_{1}, t_{2}\right\}$ is discrete. Hence, in our model, it is difficult to apply those criteria directly in order to check the strategic stability of the derived equilibria.
} 


\section{Appendix}

The following simple computation result will be used frequently in the analysis. Recall that $\gamma>1$ and $\phi>1$.

Result 1: $\frac{\phi-1}{\gamma+\phi-2}-\frac{1}{2}=\frac{(\phi-\gamma)}{2(\gamma+\phi-2)}$ and $\frac{\phi-1}{\gamma+\phi-2}-1=-\frac{(\gamma-1)}{(\gamma+\phi-2)}<0$.

\subsection{Proof of Lemma 3}

From (5), if $p \gtreqless \frac{\phi-1}{\gamma+\phi-2}, E \pi_{m}\left(t_{m}=t_{1}\right) \lesseqgtr E \pi_{m}\left(t_{m}=t_{2}\right)$. This satisfies that M's strategy is monotone increasing. Recall Result 1. Then i) if $\gamma \geq \phi, E \pi_{m}\left(t_{m}=t_{1}\right)<E \pi_{m}\left(t_{m}=t_{2}\right)$ for all $p \in\left(\frac{1}{2}, 1\right)$, ii) if $\gamma<\phi$, for $p \in\left(\frac{1}{2}, \frac{\phi-1}{\gamma+\phi-2}\right), E \pi_{m}\left(t_{m}=t_{1}\right)>E \pi_{m}\left(t_{m}=t_{2}\right)$ and for $p \in\left(\frac{\phi-1}{\gamma+\phi-2}, 1\right), E \pi_{m}\left(t_{m}=t_{1}\right)<$ $E \pi_{m}\left(t_{m}=t_{2}\right)$. Then the partially revealing separating equilibrium is supported only if $\gamma<\phi$. In this case, $\mathrm{M}$ is also indifferent to the distinction between forecasting in round 1 and round 2 at $p=\frac{\phi-1}{\gamma+\phi-2}$, which means that $x=\frac{\phi-1}{\gamma+\phi-2}$. As $f\left(x=\frac{\phi-1}{\gamma+\phi-2}\right)=\frac{(3 \gamma+3 \phi-2)(\phi-\gamma)}{(\gamma+\phi-2)}>0$, the condition that $f(x)>0$ is also satisfied. Finally, the result can be summarized as follows: If $\gamma<\phi$, for $p \in\left(\frac{1}{2}, \frac{\phi-1}{\gamma+\phi-2}\right), t_{m}=t_{1}$ and for $p \in\left(\frac{\phi-1}{\gamma+\phi-2}, 1\right), t_{m}=t_{2}$.

\subsection{Proof of Lemma 4}

We check the condition under which $\frac{3 \gamma-\phi+2}{2 \gamma+2 \phi+4} \in\left(\frac{1}{2}, 1\right)$ is satisfied. Note that $\frac{3 \gamma-\phi+2}{2 \gamma+2 \phi+4}-\frac{1}{2}=-\frac{(\phi-\gamma)}{(\gamma+\phi+2)}$ and $\frac{3 \gamma-\phi+2}{2 \gamma+2 \phi+4}-1=-\frac{(3 \phi-\gamma+2)}{2(\gamma+\phi+2)}$. So i) if $\gamma>\phi, \frac{3 \gamma-\phi+2}{2 \gamma+2 \phi+4}>\frac{1}{2}$ and if $\gamma \leq \phi, \frac{3 \gamma-\phi+2}{2 \gamma+2 \phi+4} \leq \frac{1}{2}$, ii) if $\gamma<3 \phi+2$, $\frac{3 \gamma-\phi+2}{2 \gamma+2 \phi+4}<1$ and if $\gamma \geq 3 \phi+2, \frac{3 \gamma-\phi+2}{2 \gamma+2 \phi+4} \geq 1$. Hence, a) if $\phi \geq \gamma, \frac{3 \gamma-\phi+2}{2 \gamma+2 \phi+4} \notin\left(\frac{1}{2}, 1\right)$, b) if $\phi<\gamma<3 \phi+2$, $\frac{3 \gamma-\phi+2}{2 \gamma+2 \phi+4} \in\left(\frac{1}{2}, 1\right)$ and c) if $\gamma \geq 3 \phi+2, \frac{3 \gamma-\phi+2}{2 \gamma+2 \phi+4} \notin\left(\frac{1}{2}, 1\right)$. Therefore, $x=\frac{3 \gamma-\phi+2}{2 \gamma+2 \phi+4} \in\left(\frac{1}{2}, 1\right)$ is satisfied only if $\phi<\gamma<3 \phi+2$. Then, the result can be summarized as follows: i) If $\phi<\gamma<3 \phi+2$, for $p \in\left(\frac{1}{2}, \frac{3 \gamma-\phi+2}{2 \gamma+2 \phi+4}\right), t_{m}=t_{1}$ and for $p \in\left(\frac{3 \gamma-\phi+2}{2 \gamma+2 \phi+4}, 1\right), t_{m}=t_{2}$.

\subsection{Proof of Lemma 5}

CASE 1) When $g(y)>0$

In this case, if $\mathrm{M}$ forecasts in round 1, U imitates M's forecast in round 2. Then the computation using (5) yields that if $p \gtreqless \frac{\phi-1}{\gamma+\phi-2}, E \pi_{m}\left(t_{m}=t_{1}\right) \lesseqgtr E \pi_{m}\left(t_{m}=t_{2}\right)$. However, this contradicts the conjecture that M's strategy is monotone decreasing. Hence, this case is excluded.

CASE 2) When $g(y)<0$

In this case, if $\mathrm{M}$ forecasts in round $1, \mathrm{U}$ deviates from M's forecast in round 2. Then the computation using (5) yields that if $p \gtreqless \frac{\phi-1}{\gamma+\phi-2}, E \pi_{m}\left(t_{m}=t_{1}\right) \gtreqless E \pi_{m}\left(t_{m}=t_{2}\right)$. This satisfies the condition that M's strategy is monotone decreasing. Recall Result 1. Then i) if $\gamma \geq \phi, E \pi_{m}\left(t_{m}=t_{1}\right)>E \pi_{m}\left(t_{m}=t_{2}\right)$ for all $p \in\left(\frac{1}{2}, 1\right)$, ii) if $\gamma<\phi$, for $p \in\left(\frac{\phi-1}{\gamma+\phi-2}, 1\right), E \pi_{m}\left(t_{m}=t_{1}\right)>E \pi_{m}\left(t_{m}=t_{2}\right)$ and for $p \in\left(\frac{1}{2}, \frac{\phi-1}{\gamma+\phi-2}\right)$, $E \pi_{m}\left(t_{m}=t_{1}\right)<E \pi_{m}\left(t_{m}=t_{2}\right)$. Thus, the partially revealing separating equilibrium is supported only if $\gamma<\phi$. Moreover, in that case $y=\frac{\phi-1}{\gamma+\phi-2}$ because $\mathrm{M}$ is indifferent between forecasting in round 1 and round 2 at $p=\frac{\phi-1}{\gamma+\phi-2}$. Note that $g\left(y=\frac{\phi-1}{\gamma+\phi-2}\right)=\frac{\left(\gamma-\phi+\gamma \phi-\gamma^{2}+2 \phi^{2}-2\right)}{(\gamma+\phi-2)}$. If we denote the numerator 
as $h(\phi) \equiv 2 \phi^{2}+\phi(\gamma-1)+\gamma-\gamma^{2}-2$, then the following points can be checked: a) $h(\phi)$ is a convex function, b) $h(\phi)$ attains the minimized value at $\phi=-\frac{\gamma-1}{4}<0$ and c) $h(\phi=\gamma)=2(\gamma-1)(\gamma+1)>0$. Hence, for $\phi$ such that $\gamma<\phi, h(\phi)>0$. Note that the denominator is always strictly positive because $\gamma, \phi>1$. Therefore, for $\gamma<\phi, g\left(y=\frac{\phi-1}{\gamma+\phi-2}\right)>0$ and this contradicts to the condition that $g(y)<0$. Therefore this case is excluded.

CASE 3: When $g(y)=0$

In this case, if $\mathrm{M}$ forecasts in round $1, \mathrm{U}$ is indifferent between imitating and deviating from M's forecast. From (10), if $\mathrm{U}$ imitates (or deviates from) $a_{m}$ with probability $\frac{1}{2}$, $\mathrm{M}$ is indifferent to the choice between forecasting in round 1 and round 2 for all $p \in\left(\frac{1}{2}, 1\right)$. Then, for the requirement that U's posterior belief that $p$ should be consistent, we only have to check whether $\frac{\gamma-\phi}{\gamma+\phi+2} \in\left(\frac{1}{2}, 1\right)$ for which $g\left(y=\frac{\gamma-\phi}{\gamma+\phi+2}\right)=0$. Note that $\frac{\gamma-\phi}{\gamma+\phi+2}-\frac{1}{2}=-\frac{(3 \phi-\gamma+2)}{2(\gamma+\phi+2)}$ and $\frac{\gamma-\phi}{\gamma+\phi+2}-1=-\frac{2(\phi+1)}{(\gamma+\phi+2)}<0$. So, if $\gamma \leq 3 \phi+2$, $\frac{\gamma-\phi}{\gamma+\phi+2} \notin\left(\frac{1}{2}, 1\right)$, and if $\gamma>3 \phi+2, \frac{\gamma-\phi}{\gamma+\phi+2} \in\left(\frac{1}{2}, 1\right)$. Hence, a partially revealing separating equilibrium can be supported only if $\gamma>3 \phi+2$. Therefore, the result can be summarized as follows: If $\gamma>3 \phi+2$, for $p \in\left(\frac{\gamma-\phi}{\gamma+\phi+2}, 1\right), t_{m}=t_{1}$ and for $p \in\left(\frac{1}{2}, \frac{\gamma-\phi}{\gamma+\phi+2}\right), t_{m}=t_{2}$.

\subsection{Proof of Proposition 3}

First, we check U's best response. Suppose that U observes that $t_{m}=t_{1}$. Then

$$
\begin{aligned}
E \pi_{u}\left(a_{u}=a_{m}, w \mid t_{m}=t_{1}\right) & =\int\left(\sum_{w \in\{H, L\}} \operatorname{Pr}(w \mid \theta=h) \pi_{u}\left(a_{u}=a_{m}, w\right)\right) d Z\left(p \mid t_{m}=t_{1}\right) \\
& =\int_{p \in\left(\frac{1}{2}, 1\right)}(p-(1-p)) d Z(p) \\
& =\frac{1}{2} \\
E \pi_{u}\left(a_{u} \neq a_{m}, w \mid t_{m}=t_{1}\right) & =\int\left(\sum_{w \in\{H, L\}} \operatorname{Pr}(w \mid \theta=h) \pi_{u}\left(a_{u} \neq a_{m}, w\right)\right) d Z\left(p \mid t_{m}=t_{1}\right) \\
& =\int_{p \in\left(\frac{1}{2}, 1\right)}(p(-\phi)+(1-p) \gamma) d Z(p) \\
& =\left(-\frac{1}{4}\right)(3 \phi-\gamma)
\end{aligned}
$$

Then,

$$
E \pi_{u}\left(a_{u}=a_{m}, w \mid t_{m}=t_{1}\right)-E \pi_{u}\left(a_{u} \neq a_{m}, w \mid t_{m}=t_{1}\right)=\frac{1}{4}(3 \phi-\gamma+2)
$$

Hence, U's best response in round 2 for $t_{m}=t_{1}$ is as follows: i) if $3 \phi+2>\gamma$, U imitates, ii) if $3 \phi+2<\gamma, U$ deviates, iii) if $3 \phi+2=\gamma, U$ is indifferent to both imitating and deviating from $a_{m}$.

Next, we consider M's decision on his timing of forecast.

Case 1: When $\gamma<3 \phi+2$ 
If $t_{m}=t_{1}, \mathrm{U}$ imitates $a_{m}$ in round 2. Then from (5), if $p \gtreqless \frac{\phi-1}{\gamma+\phi-2}, E \pi_{m}\left(t_{m}=t_{1}\right) \lesseqgtr E \pi_{m}\left(t_{m}=t_{2}\right)$. Recall Result 1. Then i) if $\gamma \geq \phi$, for all $p \in\left(\frac{1}{2}, 1\right), E \pi_{m}\left(t_{m}=t_{1}\right)<E \pi_{m}\left(t_{m}=t_{2}\right)$, ii) if $\gamma<\phi$, for $p \in$ $\left(\frac{\phi-1}{\gamma+\phi-2}, 1\right), E \pi_{m}\left(t_{m}=t_{1}\right)<E \pi_{m}\left(t_{m}=t_{2}\right)$ and for $p \in\left(\frac{1}{2}, \frac{\phi-1}{\gamma+\phi-2}\right), E \pi_{m}\left(t_{m}=t_{1}\right)>E \pi_{m}\left(t_{m}=t_{2}\right)$. Therefore, there is no case in which the pooling equilibrium, in which $E \pi_{m}\left(t_{m}=t_{1}\right)>E \pi_{m}\left(t_{m}=t_{2}\right)$ for $p \in\left(\frac{1}{2}, 1\right)$, is supported.

Case 2: When $\gamma>3 \phi+2$

If $t_{m}=t_{1}$, U deviates from $a_{m}$. Then from (7), if $p \gtreqless \frac{\phi-1}{\gamma+\phi-2}, E \pi_{m}\left(t_{m}=t_{1}\right) \gtreqless E \pi_{m}\left(t_{m}=t_{2}\right)$. Recall Result 1. Then i) if $\gamma \geq \phi$, for all $p \in\left(\frac{1}{2}, 1\right), E \pi_{m}\left(t_{m}=t_{1}\right)>E \pi_{m}\left(t_{m}=t_{2}\right)$, ii) if $\gamma<\phi$, for $p \in$ $\left(\frac{\phi-1}{\gamma+\phi-2}, 1\right), E \pi_{m}\left(t_{m}=t_{1}\right)>E \pi_{m}\left(t_{m}=t_{2}\right)$ and for $p \in\left(\frac{1}{2}, \frac{\phi-1}{\gamma+\phi-2}\right), E \pi_{m}\left(t_{m}=t_{1}\right)<E \pi_{m}\left(t_{m}=t_{2}\right)$. Therefore, pooling equilibrium is supported only if $\gamma \geq \phi$ and the given condition that $\gamma>3 \phi+2$ satisfies this. Hence, if $\gamma>3 \phi+2$, the pooling strategy can be supported as the equilibrium strategy.

Case 3: When $\gamma=3 \phi+2$

If $t_{m}=t_{1}, \mathrm{U}$ is indifferent between imitating and deviating from $a_{m}$. Then from (8) and (9),

$$
E \pi_{m}\left(t_{m}=t_{1}\right)-E \pi_{m}\left(t_{m}=t_{2}\right)=z(2 p-p \gamma+\phi(1-p)-1)+\frac{1}{2}(p \gamma-\phi-2 p+p \phi+1)
$$

Note that if $p \lesseqgtr \frac{\phi-1}{\gamma+\phi-2}, 2 p-p \gamma+\phi(1-p)-1 \gtreqless 0$ where $\frac{\phi-1}{\gamma+\phi-2}-\frac{1}{2}=\frac{(\phi-\gamma)}{2(\gamma+\phi-2)}$ and $\frac{\phi-1}{\gamma+\phi-2}-1=$ $-\frac{(\gamma-1)}{(\gamma+\phi-2)}<0$. So i) if $\gamma \geq \phi$, for all $p \in\left(\frac{1}{2}, 1\right), 2 p-p \gamma+\phi(1-p)-1<0$ and ii) if $\gamma<\phi$, for $p \in\left(\frac{1}{2},-\frac{\phi-1}{2-\phi-\gamma}\right), 2 p-p \gamma+\phi(1-p)-1>0$ and for $p \in\left(-\frac{\phi-1}{2-\phi-\gamma}, 1\right), 2 p-p \gamma+\phi(1-p)-1<$ 0 . Hence, when $\gamma \geq \phi$, if $z<\frac{1}{2}, E \pi_{m}\left(t_{m}=t_{1}\right)>E \pi_{m}\left(t_{m}=t_{2}\right)$ for all $p \in\left(\frac{1}{2}, 1\right)$ and if $z \geq \frac{1}{2}$, $E \pi_{m}\left(t_{m}=t_{1}\right) \leq E \pi_{m}\left(t_{m}=t_{2}\right)$ for all $p \in\left(\frac{1}{2}, 1\right)$. On the other hand, if $\gamma<\phi$, M's pooling strategy cannot be sustained. Therefore, the pooling equilibrium can be supported only if $\gamma \geq \phi$ and $z \leq \frac{1}{2}$ where $z=\operatorname{Pr}\left(a_{u}=a_{m}\right)$. Moreover, the given condition that $\gamma=3 \phi+2$ satisfies the condition that $\gamma \geq \phi$.

\subsection{Proof of Lemma 6}

Suppose that $\mathrm{M}$ forecasted in round 1. Without loss of generality, assume that $a_{m}=h$. Then, U infers that $\theta=h$. Hence, from her posterior beliefs about the true state such that $\operatorname{Pr}(w=H \mid \theta=h)=p$ and $\operatorname{Pr}(w=L \mid \theta=h)=1-p$,

$$
\begin{aligned}
E \pi_{u}\left(a_{m}, a_{u}=a_{m}, w\right) & =\sum_{w \in\{H, L\}} \operatorname{Pr}(w \mid \theta=h) \pi_{u}\left(a_{m}, a_{u}, w\right) \\
& =2 p-1 \\
E \pi_{u}\left(a_{m}, a_{u} \neq a_{m}, w\right) & =\sum_{w \in\{H, L\}} \operatorname{Pr}(w \mid \theta=h) \pi_{u}\left(a_{m}, a_{u}, w\right) \\
& =-p \phi+(1-p) \gamma
\end{aligned}
$$

(A3) is U's expected payoff when U imitates M's forecast and (A4) is the one when U deviates from M's forecast. Then,

$$
E \pi_{u}\left(a_{m}, a_{u}=a_{m}, w\right)-E \pi_{u}\left(a_{m}, a_{u} \neq a_{m}, w\right)=p(\gamma+\phi+2)-\gamma-1
$$


Hence, if $p \gtreqless \frac{\gamma+1}{\gamma+\phi+2}, E \pi_{u}\left(a_{u}=a_{m}\right) \gtreqless E \pi_{u}\left(a_{u} \neq a_{m}\right)$. Note that $\frac{\gamma+1}{\gamma+\phi+2}-\frac{1}{2}=-\frac{(\phi-\gamma)}{2(\gamma+\phi+2)}$ and $\frac{\gamma+1}{\gamma+\phi+2}-1=-\frac{(\phi+1)}{(\gamma+\phi+2)}<0$. So if $\gamma>\phi, \frac{\gamma+1}{\gamma+\phi+2} \in\left(\frac{1}{2}, 1\right)$, but if $\gamma \leq \phi, \frac{\gamma+1}{\gamma+\phi+2} \leq \frac{1}{2}$. Therefore the following result can be proposed: 1 ) if $\gamma>\phi$, for $p \in\left(\frac{1}{2}, \frac{\gamma+1}{\gamma+\phi+2}\right)$, U deviates from $a_{m}$ and for $p \in\left(\frac{\gamma+1}{\gamma+\phi+2}, 1\right)$, U imitates $\left.a_{m} .2\right)$ if $\gamma \leq \phi$, U imitates $a_{m}$ for all $p \in\left(\frac{1}{2}, 1\right)$.

\subsection{Proof of Proposition 4}

Without loss of generality, assume that $\theta=h$. Also, recall (3) which is M's expected payoff when he forecasts in round 2 .

First, suppose that $\gamma \leq \phi$. In this case, $\mathrm{M}$ knows that, if he forecasts in round 1 , U imitates $a_{m}$ for all $p \in\left(\frac{1}{2}, 1\right)$. Then from (5) if $p \gtreqless \frac{\phi-1}{\gamma+\phi-2}, E \pi_{m}\left(t_{m}=t_{1}\right) \lesseqgtr E \pi_{m}\left(t_{m}=t_{2}\right)$. Recall Result 1 . Then, if $\gamma<\phi, \frac{\phi-1}{\gamma+\phi-2} \in\left(\frac{1}{2}, 1\right)$ and if $\gamma=\phi, \frac{\phi-1}{\gamma+\phi-2}=\frac{1}{2}$. Therefore, if $\gamma<\phi$, for $p \in\left(\frac{1}{2}, \frac{\phi-1}{\gamma+\phi-2}\right)$, $E \pi_{m}\left(t_{m}=t_{1}\right)>E \pi_{m}\left(t_{m}=t_{2}\right)$ and for $p \in\left(\frac{\phi-1}{\gamma+\phi-2}, 1\right), E \pi_{m}\left(t_{m}=t_{1}\right)<E \pi_{m}\left(t_{m}=t_{2}\right)$. On the other hand, if $\gamma=\phi$, for all $p \in\left(\frac{1}{2}, 1\right), E \pi_{m}\left(t_{m}=t_{1}\right)<E \pi_{m}\left(t_{m}=t_{2}\right)$.

Second, suppose that $\gamma>\phi$. If $p \in\left(\frac{1}{2}, \frac{\gamma+1}{\gamma+\phi+2}\right)$, M knows that, if he forecasts in round $1, \mathrm{U}$ deviates from $a_{m}$. Then from (7), if $p \gtreqless \frac{\phi-1}{\gamma+\phi-2}, E \pi_{m}\left(t_{m}=t_{1}\right) \gtreqless E \pi_{m}\left(t_{m}=t_{2}\right)$. Note that $\frac{1}{2}-\frac{(\phi-1)}{\gamma+\phi-2}=$ $\frac{(\gamma-\phi)}{2(\gamma+\phi-2)}>0$ for $\gamma>\phi$, which implies that $E \pi_{m}\left(t_{m}=t_{1}\right)>E \pi_{m}\left(t_{m}=t_{2}\right)$ for all $p$. So if $p \in\left(\frac{1}{2}, \frac{\gamma+1}{\gamma+\phi+2}\right)$, $E \pi_{m}\left(t_{m}=t_{1}\right)>E \pi_{m}\left(t_{m}=t_{2}\right)$. On the other hand if $p \in\left(\frac{\gamma+1}{\gamma+\phi+2}, 1\right), \mathrm{M}$ knows that if he forecasts in round $1, \mathrm{U}$ imitates $a_{M}$. In this case, note that if $p \gtreqless \frac{\phi-1}{\gamma+\phi-2}, E \pi_{m}\left(t_{m}=t_{1}\right) \lesseqgtr E \pi_{m}\left(t_{m}=t_{2}\right)$ where $\frac{1}{2}>\frac{(\phi-1)}{\gamma+\phi-2}$ for $\gamma>\phi$. This implies that $E \pi_{m}\left(t_{m}=t_{1}\right) \lesseqgtr E \pi_{m}\left(t_{m}=t_{2}\right)$ for all $p$. Hence for $p \in\left(\frac{\gamma+1}{\gamma+\phi+2}, 1\right), E \pi_{m}\left(t_{m}=t_{1}\right)<E \pi_{m}\left(t_{m}=t_{2}\right)$.

\subsection{Proof of Proposition 6}

Consider the case in which $U$ believes that M's strategy is monotone decreasing. That is, U believes as follows: "There exists a critical value $y \in\left[\frac{1}{2}, 1\right)$ such that if $p \in(y, 1), t_{m}=t_{1}$ and if $p \in\left(\frac{1}{2}, y\right)$, $t_{m}=t_{2}$." As we are considering the case in which $\mathrm{U}$ observes that $t_{m}=t_{1}$, the case in which $y=1$ is excluded. Under this belief, if $\mathrm{U}$ observes that $t_{m}=t_{1}$, from (11) and (12), U's best response is as follows: If $y>\frac{\gamma-\phi}{\gamma+\phi+2}, U$ imitates $a_{m}$, if $y<\frac{\gamma-\phi}{\gamma+\phi+2}, U$ deviates from $a_{m}$ and if $y=\frac{\gamma-\phi}{\gamma+\phi+2}, U$ is indifferent. Note that $\frac{\gamma-\phi}{\gamma+\phi+2}-\frac{1}{2}=-\frac{(3 \phi-\gamma+2)}{2(\gamma+\phi+2)}$ and $\frac{\gamma-\phi}{\gamma+\phi+2}-1=-\frac{2(\phi+1)}{(\gamma+\phi+2)}<0$. Then, U's best response in round 2 for $t_{m}=t_{1}$ can be summarized as follows.

\section{Lemma A.1.}

For $t_{m}=t_{1}$ which is off-the-equilibrium path, if $U$ believes that $M$ 's strategy is monotone decreasing, U's best response for $t_{m}=t_{1}$ can be described as follows.

1) If $\gamma<3 \phi+2$, U imitates $a_{m}$ for all $y \in\left[\frac{1}{2}, 1\right)$.

2) If $\gamma=3 \phi+2$, $U$ imitates $a_{m}$ for $y \in\left(\frac{1}{2}, 1\right)$ and $U$ is indifferent for $y=\frac{1}{2}$.

3) If $\gamma>3 \phi+2$, U deviates from $a_{m}$ for $y \in\left[\frac{1}{2}, \frac{\gamma-\phi}{\gamma+\phi+2}\right)$, $U$ is indifferent for $y=\frac{\gamma-\phi}{\gamma+\phi+2}$ and $U$ imitates $a_{m}$ for $y \in\left(\frac{\gamma-\phi}{\gamma+\phi+2}, 1\right)$. 
Now recall the following M's decision on $t_{m} \in\left\{t_{1}, t_{2}\right\}$ against U's best response when $a_{m}$ is observable.

Case A) When $\mathrm{U}$ imitates $a_{m}$ if it is observable:

1) If $\gamma \geq \phi \Longrightarrow E \pi_{m}\left(t_{m}=t_{1}\right)<E \pi_{m}\left(t_{m}=t_{2}\right)$ for all $p \in\left(\frac{1}{2}, 1\right)$

2) If $\gamma<\phi \Longrightarrow\left\{\begin{array}{l}E \pi_{m}\left(t_{m}=t_{1}\right)>E \pi_{m}\left(t_{m}=t_{2}\right) \text { for } p \in\left(\frac{1}{2}, \frac{\phi-1}{\gamma+\phi-2}\right) \\ E \pi_{m}\left(t_{m}=t_{1}\right)<E \pi_{m}\left(t_{m}=t_{2}\right) \text { for } p \in\left(\frac{\phi-1}{\gamma+\phi-2}, 1\right)\end{array}\right.$

Case B) When $\mathrm{U}$ deviates from $a_{m}$ if it is observable.

1) If $\gamma \geq \phi \Longrightarrow E \pi_{m}\left(t_{m}=t_{1}\right)>E \pi_{m}\left(t_{m}=t_{2}\right)$ for all $p \in\left(\frac{1}{2}, 1\right)$

2) If $\gamma<\phi \Longrightarrow \begin{cases}E \pi_{m}\left(t_{m}=t_{1}\right)<E \pi_{m}\left(t_{m}=t_{2}\right) & \text { for } p \in\left(\frac{1}{2}, \frac{\phi-1}{\gamma+\phi-2}\right) \\ E \pi_{m}\left(t_{m}=t_{1}\right)>E \pi_{m}\left(t_{m}=t_{2}\right) & \text { for } p \in\left(\frac{\phi-1}{\gamma+\phi-2}, 1\right)\end{cases}$

Case C) When $\mathrm{U}$ is indifferent between imitating and deviating from $a_{m}$ if it is observable:

1) i) if $\gamma>\phi$, then for all $p \in\left(\frac{1}{2}, 1\right)$ or ii) if $\gamma<\phi$ and $p \in\left(\frac{\phi-1}{\gamma+\phi-2}, 1\right), z \lesseqgtr \frac{1}{2} \Longrightarrow E \pi_{m}\left(t_{m}=t_{1}\right) \gtreqless$ $E \pi_{m}\left(t_{m}=t_{2}\right)$.

2) If $\gamma<\phi$ and $p \in\left(\frac{1}{2}, \frac{\phi-1}{\gamma+\phi-2}\right), z \gtreqless \frac{1}{2} \Longrightarrow E \pi_{m}\left(t_{m}=t_{1}\right) \gtreqless E \pi_{m}\left(t_{m}=t_{2}\right)$.

Now we check whether M's pooling strategy, " $t_{m}=t_{2}$ for all $p \in\left(\frac{1}{2}, 1\right)$ ", can be sustained as the equilibrium strategy or not. Recall Lemma A.1.

Case 1) When $\gamma<3 \phi+2$ : In this case, for all $y \in\left[\frac{1}{2}, 1\right)$, U imitates $a_{m}$. Then, from Case A, the given pooling strategy is sustained only if $\phi \leq \gamma<3 \phi+2$.

Case 2) When $\gamma=3 \phi+2$ : First, if $y \in\left(\frac{1}{2}, 1\right)$, U imitates $a_{m}$. Then, from Case A, the given pooling strategy is sustained if $\gamma=3 \phi+2$. Second, if $y=\frac{1}{2}$, $\mathrm{U}$ is indifferent to either imitation or deviation $a_{m}$. Then, from Case $\mathrm{C}$, the given pooling strategy can be sustained only if $z>\frac{1}{2}$.

Case 3) When $\gamma>3 \phi+2$ : First, if $y \in\left[\frac{1}{2}, \frac{\gamma-\phi}{\gamma+\phi+2}\right)$, U deviates from $a_{m}$. Then, from Case B, the given pooling strategy cannot be sustained. Second, if $y=\frac{\gamma-\phi}{\gamma+\phi+2}$, as U is indifferent, from Case C, the given pooling strategy can be sustained only if $z>\frac{1}{2}$. Finally, if $y \in\left(\frac{\gamma-\phi}{\gamma+\phi+2}, 1\right)$, U imitates $a_{m}$. Then, from Case A, the given pooling strategy can be sustained.

This completes the proof.

\subsection{Proof of Corollary 3}

In the following, we denote each player's expected payoff and each equilibrium as follows:

- $E \pi_{i}^{P 1}$ : Player $i$ 's expected payoff in the pooling equilibrium where $\theta$ is revealed for all $p \in\left(\frac{1}{2}, 1\right)$.

- $E \pi_{i}^{P 2}$ : Player $i$ 's expected payoff in the pooling equilibrium where $\theta$ is not revealed for all $p \in\left(\frac{1}{2}, 1\right)$.

- $E \pi_{i}^{S}$ : Player $i$ 's expected payoff in the partially revealing separating equilibrium.

- PE1 : The pooling equilibrium where $\theta$ is revealed for all $p \in\left(\frac{1}{2}, 1\right)$.

- PE2 : The pooling equilibrium where $\theta$ is not revealed at all for all $p \in\left(\frac{1}{2}, 1\right)$.

- SE : The partially revealing separating equilibrium. 
Case 1: When $\gamma>3 \phi+2$

In this case, PE1, PE2 and SE exist.

First, we show that PE1 dominates SE. Consider PE1. In this equilibrium, $\mathrm{M}$ forecasts in round 1 for all $p \in\left(\frac{1}{2}, 1\right)$ and $\mathrm{U}$ deviates from $a_{m}$ if observable. Hence, from (6), $E \pi_{m}^{P 1}=p \gamma-\phi+p \phi$. Also from (12), $E \pi_{u}^{P 1}=\left.E \pi_{u}\left(a_{u} \neq a_{m}, w \mid t_{m}=t_{1}\right)\right|_{y=\frac{1}{2}}=\left(-\frac{1}{4}\right)(3 \phi-\gamma)$. Next consider SE. As it is actually a mixed strategy equilibrium, M's expected payoffs are same regardless of the value of $p$. From (9), $E \pi_{m}^{S}=E \pi_{m}\left(t_{m}=t_{1}\right)=\frac{1}{2}(4 p-\phi+p \gamma+p \phi-2)$. Also from (11) or (12) where $y=\frac{\gamma-\phi}{\gamma+\phi+2}$, $E \pi_{u}^{S}=\frac{\gamma-\phi}{\gamma+\phi+2}$. Then,

$$
E \pi_{u}^{P 1}-E \pi_{u}^{S}=-\frac{(\gamma+\phi)(3 \phi-\gamma+2)}{4(\gamma+\phi+2)}>0
$$

because our condition is that $\gamma>3 \phi+2$. Also

$$
E \pi_{m}^{P 1}-E \pi_{m}^{S}=\frac{1}{2}(p \gamma-\phi-4 p+p \phi+2)
$$

Then $p \gtrless \frac{\phi-2}{\gamma+\phi-4} \Longrightarrow E \pi_{m}^{P} \gtrless E \pi_{m}^{S}$. Note that $\frac{\phi-2}{\gamma+\phi-4}-\frac{1}{2}=\frac{(\phi-\gamma)}{2(\gamma+\phi-4)}<0$ because our condition is that $\gamma>3 \phi+2>\phi$. So for all $p \in\left(\frac{1}{2}, 1\right), E \pi_{m}^{P}>E \pi_{m}^{S}$. Therefore, PE1 dominates SE.

Second, we show that PE1 also dominates PE2. In PE2, M always delays his forecast and U should randomize his forecast. Then from (3), $E \pi_{m}^{P 2}\left(t_{m}=t_{2}\right)=\frac{1}{2}(2 p-\phi+p \gamma+p \phi-1)$. In the case of U's expected payoff, if we assume that $a_{u}=h$,

$$
\begin{aligned}
E \pi_{u}^{P 2} & =\int \sum_{w \in\{H, L\}} \sum_{\theta \in\{h, l\}} \operatorname{Pr}(w, \theta) \pi_{u}\left(a_{u}=h, a_{m}=\theta, w\right) d Z\left(p \mid t_{m}=t_{2}\right) \\
& =\int_{p \in\left(\frac{1}{2}, 1\right)}\left(-\frac{1}{2}\right)(p \gamma-\gamma-2 p+p \phi+1) d Z(p) \\
& =\left(-\frac{1}{8}\right)(3 \phi-\gamma-2)
\end{aligned}
$$

Then

$$
E \pi_{m}^{P 2}-E \pi_{m}^{P 1}=\left(-\frac{1}{2}\right)(p \gamma-\phi-2 p+p \phi+1)<0
$$

because $p \gtrless \frac{\phi-1}{\gamma+\phi-2} \Longrightarrow E \pi_{m}^{P 2}\left(t_{m}=t_{2}\right) \lessgtr E \pi_{m}^{P 1}$ where $\frac{\phi-1}{\gamma+\phi-2}-\frac{1}{2}=\frac{(\phi-\gamma)}{2(\gamma+\phi-2)}<0$ for $\gamma>\phi$. Also

$$
E \pi_{u}^{P 2}-E \pi_{u}^{P 1}=\frac{1}{8}(3 \phi-\gamma+2)<0
$$

because our condition is that $\gamma>3 \phi+2$.

Finally, if $\gamma>3 \phi+2$, PE1 dominates other equilibria in terms of expected payoff.

Case 2: When $\gamma=3 \phi+2$

In this case, there exist PE1 and PE2. In PE1, M forecasts in round 1 for all $p \in\left(\frac{1}{2}, 1\right)$ and $\mathrm{U}$ deviates from $a_{m}$ if observable with probability $1-z \geq \frac{1}{2}$ where $1-z=\operatorname{Pr}\left(a_{u} \neq a_{m}\right)$. From $(8), E \pi_{m}^{P 1}=$ $z(p-(1-p))+(1-z)(p \gamma-(1-p) \phi)$. Here it can be checked that $\frac{\partial\left(E \pi_{m}^{P 1}\right)}{\partial z}=-(p \gamma-\phi-2 p+p \phi+1)<0$ for $\gamma=3 \phi+2>\phi$. Hence the value of $E \pi_{m}\left(t_{m}=t_{1}\right)$ is minimized when $z=\frac{1}{2}$ and the minimized value is $\left.E \pi_{m}\left(t_{m}=t_{1}\right)\right|_{z=\frac{1}{2}}=\frac{1}{2}(2 p-\phi+p \gamma+p \phi-1)$. In PE2, it was already checked (from Case 1$)$ that $E \pi_{m}^{P 2}\left(t_{m}=t_{2}\right)=\frac{1}{2}(2 p-\phi+p \gamma+p \phi-1)$. So

$$
E \pi_{m}^{P 2} \leq E \pi_{m}^{P 1}
$$


for $z \leq \frac{1}{2}$.

Next, in PE1 $E \pi_{u}^{P 1}=\frac{1}{2}$ from (A1) or (A2). Also in PE2, $\left.E \pi_{u}^{P 2}\right|_{\gamma=3 \phi+2}=\left(-\frac{1}{8}\right)(3 \phi-\gamma-2)=\frac{1}{2}$. Then

$$
E \pi_{u}^{P 2}=E \pi_{u}^{P 1}
$$

So if $\gamma=3 \phi+2$, PE1 (weakly) dominates PE2 in terms of expected payoff.

Case 3: When $\phi<\gamma<3 \phi+2$

In this case, there exist SE and PE2. Here, SE is actually a mixed strategy equilibrium in which each player should earn the same expected payoff regardless of his strategy. From (9), $E \pi_{m}^{S}=\frac{1}{2}(2 p-\phi+p \gamma+p \phi-1)$. Also from (1) or (2), $E \pi_{u}^{S}=\left.E \pi_{u}\left(a_{u}=a_{m}, w \mid t_{m}=t_{1}\right)\right|_{x=\frac{3 \gamma-\phi+2}{2 \gamma+2 \phi+4}}=\frac{\gamma-\phi}{\gamma+\phi+2}$. In PE2, from Case 1, $E \pi_{m}^{P 2}=\frac{1}{2}(2 p-\phi+p \gamma+p \phi-1)$ and $\left.E \pi_{u}^{P 2}\right|_{\gamma=3 \phi+2}=\left(-\frac{1}{8}\right)(3 \phi-\gamma-2)=\frac{1}{2}$. Then

$$
E \pi_{m}^{P 2}=E \pi_{m}^{S}
$$

and

$$
E \pi_{u}^{P 2}-E \pi_{u}^{S}=-\frac{(\gamma+\phi-2)(3 \phi-\gamma+2)}{8(\gamma+\phi+2)}<0
$$

because our condition is that $\gamma<3 \phi+2$. So if $\phi<\gamma<3 \phi+2$, SE (weakly) dominates PE2 in terms of expected payoff. 


\section{References}

Avery, C.N., Chevalier, J., "Herding over the career.", Economics Letters, Vol. 63 (1999), pp. 327-333.

Banerjee, A.V., "A Simple-model of herd behavior.", Quarterly Journal of Economics, Vol. 107 (1992), pp. $797-817$.

Bikhchandani, S., Hirshleifer, D., Welch, I., "A theory of fads, fashion, custom and cultural change as informational cascades.", Journal of Political Economy, Vol. 100 (1992), pp. 992-1026.

Clarke, R. "Collusion and the incentives for information sharing." Bell Journal of Economics, Vol. 14 (1983), pp. 383-394.

Conner, K.R., "Obtaining Strategic Advantage from Being Imitated: When Can Encouraging "Clones" pay?.", Management Science, Vol. 41. (1995), pp. 209-225.

Conner, K.R. and Rumelt, R.P., "Software piracy, an analysis of protection strategies." Management Science, Vol. 37 (1991), pp. 125-139.

Creane, A., "Risk and revelation: Changing the value of information." Economica, Vol. 65 (1998), pp. $247-261$.

De Fraja, G., "Strategic spillovers in patent races.", International Journal of Industrial Organization, Vol. 11 (1993), pp. 139-146.

Effinger, M.R., Polborn, M.K., "Herding and anti-herding: a model of reputational differentiation.", European Economic Review Vol. 45 (2001), pp. 385-403.

Fried, D. "Incentives for information production and disclosure in a duopolistic environment." Quarterly Journal of Economics, Vol. 99 (1984), pp. 367-381.

Frisell, L., "On the Interplay of Informational Spillovers and Payoff Externalities.", RAND Journal of Economics, Vol 34 (2003), pp. 582-592.

Gal-Or, E. "Information sharing in oligopoly." Econometrica, Vol. 53 (1985), pp. 329-343.

Gal-Or, E. "Information transmission - Cournot and Bertrand equilibria." Review of Economic Studies, Vol. 53 (1986), pp. 85-92.

Gul, F. and Lundholm, R., "Endogenous timing and the clustering of agents' decisions." Journal of Political Economy, Vol. 103 (1995), pp. 1039-1066.

Katsoulacos, Y. and Ulph, D., "Endogenous spillovers and the performance of research joint ventures.", Journal of Industrial Economics, Vol. XLVI (1998), pp. 333-357.

Levy, G., "Anti-herding and strategic consultation.", European Economic Review Vol. 48 (2004), pp. 503-525. 
Li, L. "Cournot Oligopoly with information sharing." RAND Journal of Economics, Vol. 16 (1985), pp. 521-536.

Mailath, G.J., "Endogenous sequencing of firm decisions." Journal of Economic Theory, Vol. 59 (1993), pp. 169-182.

Milgrom, P.R., "Good news and bad news: representation theorems and applications." Bell Journal of Economics, Vol. 12 pp. 380-391.

Milgrom, P.R. and Roberts, J., "Relying on the information of interested parties.", RAND Journal of Economics, Vol. 17, pp 18-32.

Okuno-Fujiwara, M., Postlewaite, A. and Suzumura, K., "Strategic information revelation.", Review of Economic Studies, Vol. 57 (1990), pp. 25-47.

Perez-Castrillo, J.D. and Sandonis, J., "Disclosure of know-how in research joint ventures.", International Journal of Industrial Organization, Vol. 15 (1996), pp. 51-75.

Poyago-Theotoky, J., "A note on endogenous spillovers in a non-tournament R\&D duopoly.", Review of Industrial Organization, Vol. 15 (1999), pp. 253-262.

Raith, M. "A general model of information sharing in oligopoly." Journal of Economic Theory, Vol. 71 (1996), pp. 260-288.

Rosenkranz, S., "To reveal or not to reveal: know-how disclosure and joint ventures in procurement auctions.", Journal of Institutional and Theoretical Economics, Vol. 157 (2001), pp. 555-567.

Scharfstein, D.S., Stein, J.C., "Herd behavior and investment.", American Economic Review Vol. 80 (1990), pp. 465-479.

Shapiro, C. "Exchange of cost information in oligopoly." Review of Economic Studies, Vol. 87 (1973), pp. $355-374$.

Trueman, B., "Analyst forecasting and herding behavior.", Review of Financial Studies Vol. 7 (1994), pp. $97-124$.

Vives, X. "Duopoly information equilibrium: Cournot and Bertrand." Journal of Economic Theory, Vol. 34 (1984), pp. 71-94.

Vives, X. "Trade association disclosure rules, incentives to share information, and welfare." RAND Journal of Economics, Vol. 21 (1990), pp. 409-430.

Ziv, A. "Information sharing in oligopoly: the truth-telling problem." RAND Journal of Economics, Vol. 24(3) (1993), pp.455-465. 